Pacific Journal of Mathematics

REGULAR ELEMENTS IN AN ORDERED SEMIGROUP 


\section{REGULAR ELEMENTS IN AN ORDERED SEMIGROUP}

\section{TôRU SAITô}

Introduction. In the abstract theory of semigroups, the begriff of regular element was first introduced by Thierrin [11] as a generalization in the semigroup theory of the begriff of inverse element in the group theory. And this begriff of regular element has been effectively used in the ideal theory of semigroups, for example, in Miller and Clifford [5]. But the structure of regular semigroups, that is, semigroups in which all the elements are regular, is complicated and until now very little was known about it. Inverse semigroup is an important sort of regular semigroups, whose structure was completely determined (Preston [7], [8]).

Ordered semigroups have been studied by several authors, for example, Alimov [1] and Clifford [2]. However, as far as we know, none discussed systematically ordered semigroups in our general sense (cf. $\S 1$ ). In the previous paper [10], we characterized ordered idempotent semigroups, that is, ordered semigroups in which all the elements are idempotent. In the continuation of our investigation of ordered semigroups, in this note, we concern essentially with ordered regular semigroups.

Main purpose of this note is to give a catalog of all possible types of subsemigroups generated by regular pairs of ordered semigroups. The subsemigroups of an ordered semigroup $S$ generated by regular pairs are the analogs of the cyclic subgroups of a group, in fact reduce to exactly these when $S$ is a group. It therefore should be useful in the study of ordered regular semigroups to have catalog of them available. A list of 39 ordered semigroups, each generated by a (non-idempotent) regular pair, is given in this note, and it is shown that every such ordered semigroup is (order-and-product) isomorphic with one of these. Theorems 3,4 and 5 serve as an index to this catalog.

Moreover, this note contains the following by-products which seem to be interesting:

(a) the set of idempotents of an ordered semigroup $S$ is a subsemigroup of $S$ (Corollary of Lemma 1);

(b) any regular conjugate of an idempotent of an ordered semigroup $S$ is idempotent (Theorem 1);

(c) the set of regular elements of an ordered semigroup $S$ is a subsemigroup of $S$ (Corollary 2 of Lemma 5);

(d) a regular element of finite order of an ordered semigroup $S$

Received May 23, 1962. 
can have order only 1 or 2 Theorem 2 .

Finally we remark that, even though the subsemigroup of a semigroup $S$ generated by a regular pair need not be regular in general, it is regular if $S$ is ordered.

In $\S 1$, we give some definitions and some elementary results in preparation of the following discussion. In $\$ \S 2-5$, we discuss the case when a regular pair is of finite order, while in $\$ \S 6-9$, we discuss the case when it is of infinite order. In the final $\S 10$, we remark some applications in special ordered semigroups.

1. Preliminaries. We denote by $S$ an ordered semigroup, that is, a semigroup $S$ with a simple order $<$ which satisfies the following condition:

$$
\text { for } x, y, z \in S, x<y \text { implies } x z \leqq y z \text { and } z x \leqq z y \text {. }
$$

If two elements $x$ and $y$ of $S$ generate the same principal left ideal, then we write $x \equiv y(L)$, while if $x$ and $y$ generate the same principal right ideal, then we write $x \equiv y(R)$. We write $x \equiv y(D)$ if there exists an element $z$ of $S$ such that $x \equiv z(L)$ and $z \equiv y(R)$. As is well-known, these relations are equivalence relation (Green [3]). An element $x$ of $S$ is called regular if there exists an element $y$ of $S$ such that

$$
x y x=x \text { and } y x y=y
$$

(Miller and Clifford [5]). When a pair $(x, y)$ of elements of $S$ satisfy (2), $(x, y)$ is called a regular pair and $y$ is called a regular conjugate of $x$. As is easily seen by (2), for every regular pair $(x, y)$, both $x y$ and $y x$ are idempotents. An element $x$ of $S$ is called positive if $x^{2}>x$, while $x$ is called negative if $x^{2}<x$. For an element $x$ of $S$, the number of distinct natural powers of $x$ is called the order of $x$ (Clifford [2]). If $x$ is an element of finite order $n$, then $n$ is the minimal natural number such that $x^{n}=x^{n+1}$. Evidently $x$ is of order 1 if and only if $x$ is idempotent. The set of all idempotents of $S$ is denoted by $E$. For an ordered semigroup $S$, we call the multiplicative dual or, simply, dual of $S$ the ordered semigroup constructed from $S$ by interchanging the order of multiplication but by preserving the order of $S$. An element $z$ of $S$ is said to lie between $x$ and $y$, if either $x \leqq z \leqq y$ or $y \leqq z \leqq x$, while $z$ is said to lie between $x$ and $y$ in the strict sense, if either $x<z<y$ or $y<z<x$.

LEMMA 1. If $x$ and $y$ are nonnegative, then, $x y$ is nonnegative. If $x$ and $y$ are non-positive, then $x y$ is non-positive.

Proof. For nonnegative $x$ and $y$, if $x \leqq y$, then $x y \leqq x^{3} y \leqq(x y)^{2}$, 
and, if $y \leqq x$, then $x y \leqq x y^{3} \leqq(x y)^{2}$. The second assertion can be proved similarly.

Corollary. The set $E$ of all idempotents of $S$, if it is nonvoid, is a subsemigroup of $S$.

LEMMA 2. If $x$ is nonnegative, $y$ is non-positive and $x \leqq y$, then both $x y$ and $y x$ are idempotents which lie between $x$ and $y$.

Proof.

$$
x y \leqq x^{3} y \leqq(x y)^{2} \leqq x y^{3} \leqq x y,
$$

and so $x y$ is idempotent. Moreover

$$
x \leqq x^{2} \leqq x y \leqq y^{2} \leqq y .
$$

With respect to the order in $S$, the subsemigroup $E$ is clearly an ordered semigroup, which plays an important role in the following discussion. As is easily seen, for $g, h \in E$,

$$
\begin{aligned}
& g \equiv h(L) \text { in } S \text { if and only if } g h=g \text { and } h g=h, \\
& g \equiv h(R) \text { in } S \text { if and only if } g h=h \text { and } h g=g .
\end{aligned}
$$

Hence

$$
g \equiv h(L) \text { and } g \equiv h(R) \text { in } S \text { if and only if } g=h .
$$

By (3) and (4), for elements of $E, L$-equivalence and $R$-equivalence in $E$ coincide with $L$-equivalence and $R$-equivalence in $S$, respectively. However, for $D$-equivalence, such a situation does not occur. Of course, for $g, h \in E, g \equiv h(D)$ in $E$ implies $g \equiv h(D)$ in $S$, but the converse is not always true. (The semigroup $J$ in $\S 8$ will offer a counter-example.) The $D$-equivalence in $E$ is denoted by $D_{B}$-equivalence.

LEMma 3. If $g, h \in E$ and $g \leqq h$, then the following conditions are equivalent to each other:
(a) $g h \leqq h g$,
(b) $g h g=g h$,
(c) $h g h=h g$,
(d) $g h \equiv h g(L)$.

Proof. (a) implies (b), for

$$
g h=g(g h) \leqq g h g \leqq(g h) h=g h .
$$

(b) implies (c), for $h g=(h g)(h g)=h g h$. Similarly (c) implies (b). Hence if (c) holds, then both (b) and (c) hold, and so we obtain (d). Finally (d) implies (a), for, by (3), $g h=(g h)(h g)=g h g$, and so by 
Lemma $2, g \leqq g h=g h g \leqq h g \leqq h$.

LEMma 3 . If $g, h \in E$ and $g \leqq h$, then the following conditions are eqvivalent to each other:
(a) $h g \leqq g h$,
(b) $h g h=g h$,
(c) $g h g=h g$,
(d) $g h \equiv h g(R)$

CoRollaRY. $g h \equiv h g\left(D_{E}\right)$ for every $g, h \in E$.

Ordered idempotent semigroups are studied in our previous paper [10], from which we mention one more lemma without proof.

LemmA 4. Each $D_{E}$-equivalence class in $E$ consists of either only one L-equivalence class in $E$. or only one R-equivalence class in $E$.

A $D_{E}$-equivalence class, if it consists of only one $L$-equivalence class in $E$, is called $L$-typed, while, if it consists of only one $R$ equivalence class, it is called $R$-typed. A regular pair $(x, y)$ of $S$ is called $L$-typed, if the $D_{E}$-equivalence class which contains $(x y)(y x)$ is $L$-typed. By Corollary of Lemma 3 , a regular pair $(x, y)$ is $L$-typed if and only if the $D_{E}$-equivalence class which contains $(y x)(x y)$ is $L$ typed. An R-typed regular pair is defined similarly. A regular pair $(x, y)$ is said to be of order $n$, if both $x$ and $y$ are elements of order $n$. A regular pair of order 1 is also called an idempotent regular pair.

2. Idempotent regular pair. In this section, we give a theorem which characterizes idempotent regular pairs.

THEOREM 1. (a) For a regular pair $(x, y)$ of $S, x$ is idempotent if and only if $y$ is idempotent. $h\left(D_{E}\right)$.

(b) For $g, h \in E,(g, h)$ is a regular pair if and only if $g \equiv$

Proof. (a) Suppose that $(x, y)$ is a regular pair and that $x$ is an idempotent. Then $y=y x y=(y x)(x y)$ is an idempotent, by Corollary of Lemma 1 . (b) First suppose that $(g, h)$ is an idempotent regular pair and that $g \leqq h$. By (2),

$$
g \equiv h g(L), \quad h \equiv g h(L), \quad g \equiv g h(R), \quad h \equiv h g(R) .
$$

If $g h \leqq h g$, then, by Lemma $3, h g \equiv g h(L)$, and so $g \equiv h(L)$. If $h g \leqq g h$, then we obtain $g \equiv h(R)$ similarly. Next suppose that $g \equiv h\left(D_{E}\right)$. Then, by Lemma 4 , either $g \equiv h(L)$ or $g \equiv h(R)$. If $g \equiv h(L)$, then 


$$
g h g=(g h) g=g^{2}=g, \quad h g h=(h g) h=h^{2}=h,
$$

and so $(g, h)$ is a regular pair. In the case when $g \equiv h(R)$, we obtain the same result by a similar way.

3. Regular pair of finite order. In this section, we study regular pairs of finite order. But, first of all, we give some lemmas about general regular pairs which are necessary also for coming sections.

Lemma 5. For two regular pairs $(x, y)$ and $(z, w),(x z, w y)$ is a regular pair.

Proof. By Corollary of Lemma 1, both yxzw and zwyx are idempotent. Hence

$$
\begin{aligned}
& (x z)(w y)(x z)=(x y x) z w y x(z w z)=x(y x z w) z=x z, \\
& (w y)(x z)(w y)=(w z w) y x z w(y x y)=w(z w y x) y=w y .
\end{aligned}
$$

COROLLARY 1. If $(x, y)$ is a regular pair, then, for every natural number $n,\left(x^{n}, y^{n}\right)$ is a regular pair.

COROLlary 2. The set of all regular elements of $S$, if it is nonvoid, is a subsemigroup of $S$.

Lemma 6. If $(p, q)$ is a regular pair such that $q \leqq p$, then $q$ is non-positive and $p$ is nonnegative.

Proof. $q^{3} \leqq q p q=q$ and $p=p q p \leqq p^{3}$, from which the lemma follows immediately.

LEMma 7. Let $(p, q)$ be a regular pair such that $q \leqq p$ and $q p \leqq p q$. Then the following six conditions are equivalent to each other:
(a) $p q^{2}=p q^{2} p$,
(b) $q^{2} p=q^{2}$,
(c) $q^{2}=q^{3}$,
(d) $q p^{2}=q p^{2} q$,
(e) $p^{2} q=p^{2}$,
(f) $p^{2}=p^{3}$.

Moreover, these conditions imply

(g) $(q p)(p q) \equiv q^{2} \equiv p^{2} \equiv(p q)(q p)(L)$.

Proof. (a) implies (b), for $q^{2} p=q p q^{2} p=q p q^{2}=q^{2}$. (b) implies (c), for $q^{2} p^{2}=q^{2} p=q^{2}$ and so $q^{2}$ is an idempotent, by Corollary 1 of Lemma 5. (c) implies (a), for 


$$
p q^{2}=p q^{3} \leqq p q^{2} p=p q^{2}(q p) \leqq p q^{2}(p q)=p q^{2} .
$$

Similarly the conditions (d), (e) and (f) are equivalent to each other. Now (c) implies (f), for, by Theorem 1 and Corollary 1 of Lemma 5, $\left(p^{2}, q^{2}\right)$ is an idempotent regular pair. Similarly (f) implies (c). This proves the first half of the lemma. Next suppose that these conditions hold. Then

$$
\begin{aligned}
\left(q p^{2} q\right) q^{2} & =\left(\left(q p^{2} q\right) q\right) q=q p^{2} q, & q^{2}\left(q p^{2} q\right) & =\left(\left(q^{3} p\right) p\right) q=q^{2}, \\
q^{2} p^{2} & =\left(q^{2} p\right) p=q^{2}, & p^{2} q^{2} & =\left(p^{2} q\right) q=p^{2}, \\
p^{2}\left(p q^{2} p\right) & =\left(\left(p^{3} q\right) q\right) p=p^{2}, & \left(p q^{2} p\right) p^{2} & =\left(\left(p q^{2} p\right) p\right) p=p q^{2} p .
\end{aligned}
$$

Hence (g) holds.

LEMMA 7'. Let $(p, q)$ be a regular pair such that $q \leqq p$ and $p q \leqq q p$. Then the following six conditions are equivalent to each other:
(a) $q^{2} p=p q^{2} p$,
(b) $p q^{2}=q^{2}$,
(c) $q^{2}=q^{3}$,
(d) $p^{2} q=q p^{2} q$,
(e) $q p^{2}=p^{2}$,
(f) $p^{2}=p^{3}$.

Moreover, these conditions imply

(g) $(q p)(p q) \equiv q^{2} \equiv p^{2} \equiv(p q)(q p)(R)$.

CoRollary. For a regular pair $(x, y), x$ is an element of order 2 if and only if $y$ is of order 2.

THEOREM 2. If $(x, y)$ is a regular pair such that either $x$ or $y$ is an element of finite order, then $(x, y)$ is a regular pair of order either 2 or 1.

Proof. By Theorem 1 and Corollary of Lemma 7, it suffices to show that if $x$ is an element of finite order, then $x$ is of order at most 2. Here we prove this assertion only in the case when $x \leqq y$ and $x y \leqq y x$. Then, by Lemma $6, x$ is non-positive. Now suppose it were true that $x^{n-1}>x^{n}=x^{n+1}$ for a natural number $n \geqq 3$. Then $x x^{n-2}=x^{n-1}>x^{n}=x y x^{n}$ and so $x^{n-2}>y x^{n}$. Hence $x^{n-1} \geqq y x^{n+1}$. On the other hand,

$$
x^{n-1}=x y x^{n-1} \leqq(y x) x^{n-1}=y x^{n}=y x^{n+1} .
$$

Hence $x^{n-1}=y x^{n+1}$. Then we would have

$$
x^{n}=x^{n-1} x=y x^{n+2}=y x^{n+1}=x^{n-1},
$$

which is a contradiction. 
4. Ordered $T$-semigroups. In this section, we give some examples of ordered semigroups each of which has a regular element of order 2 .

EXAMPLe 1. We denote by $T_{1 L}$ the system consisting of eight elements ordered by

$$
s<q<e<t<v<f<p<u
$$

and with the following multiplication table:

\begin{tabular}{l|llllllll} 
& $s$ & $q$ & $e$ & $t$ & $v$ & $f$ & $p$ & $u$ \\
\hline$s$ & $s$ & $s$ & $s$ & $s$ & $s$ & $s$ & $s$ & $s$ \\
$q$ & $s$ & $s$ & $s$ & $s$ & $s$ & $q$ & $e$ & $t$ \\
$e$ & $s$ & $q$ & $e$ & $t$ & $t$ & $t$ & $t$ & $t$ \\
$t$ & $t$ & $t$ & $t$ & $t$ & $t$ & $t$ & $t$ & $t$ \\
$v$ & $v$ & $v$ & $v$ & $v$ & $v$ & $v$ & $v$ & $v$ \\
$f$ & $v$ & $v$ & $v$ & $v$ & $v$ & $f$ & $p$ & $u$ \\
$p$ & $v$ & $f$ & $p$ & $u$ & $u$ & $u$ & $u$ & $u$ \\
$u$ & $u$ & $u$ & $u$ & $u$ & $u$ & $u$ & $u$ & $u$
\end{tabular}.

It can be verified that this system $T_{1 L}$ is an ordered semigroup.

EXAMPLE 2. We denote by $T_{2 L}$ the system arising from $T_{1 L}$ by identifying $t$ and $v$. Clearly this identification is possible, and the constructed system $T_{2 L}$ is an ordered semigroup.

EXAMPLE 3. We denote by $T_{1 R}$ the ordered semigroup which is multiplicative dual to $T_{1 L}$. Thus the multiplication table of $T_{1 R}$ is symmetric in the main diagonal to the table (6).

ExAmple 4 . We denote by $T_{2 R}$ the ordered semigroup which is dual to $T_{2 L}$.

In each of these four ordered semigroups, $(p, q)$ is a regular pair of order 2 with negative $q$ and positive $p$. In $T_{1 L}$ and $T_{2 L},(p, q)$ is $L$-typed, while, in $T_{1 R}$ and $T_{2 R}$, it is $R$-typed. The ordered semigroups $T_{1 L}, T_{2 L}, T_{1 R}$ and $T_{2 R}$ are called ordered $T$-semigroups. Ordered $T$ semigroups $T_{1 L}$ and $T_{2 L}$ are called $L$-typed, while $T_{1 R}$ and $T_{2 R}$ are called $R$-typed.

5. Regular pair of order 2. In this section, we characterize the subsemigroup generated by a regular pair of order 2 . 
LEMMA 8. If $(x, y)$ is a non-idempotent regular pair, then both $x y$ and $y x$ lie between $x$ and $y$ in the strict sense.

Proof. Suppose that $x \leqq y$. Then, by Lemma 6 and Theorem 1, $x$ is negative and $y$ is positive. Now we suppose that $x \geqq x y$ were true. Then, by Lemma $2, x=(x y) x$ would be an idempotent, which is a contradiction. Hence $x<x y$. The remaining assertions can be proved similarly.

In the rest of this section, we assume that $(p, q)$ is a regular pair of order 2 such that $q \leqq p$, and set

$$
\begin{array}{lll}
s=q^{2}, & u=p^{2}, & e=\min \{p q, q p\}, \\
f=\max \{p q, q p\}, & t=\min \{e f, f e\}, & v=\max \{e f, f e\} .
\end{array}
$$

First we suppose that $q p \leqq p q$. Then, by Lemma 7, $(q p)(p q) \equiv$ $(p q)(q p)(L)$, and so, by Lemma 3, $(q p)(p q) \leqq(p q)(q p)$. Hence

$$
e=q p, \quad f=p q, \quad t=e f, \quad v=f e .
$$

By Lemma 8, $s=q^{2}<q<e$ and $f<p<p^{2}=u$. Moreover, by Lemma 7, $p t=p^{2} q=p^{2}>p=p e$ and $q v=q^{2} p=q^{2}<q=q f$, and so $t>e$ and $v<f$. Thus

$$
s<q<e<t \leqq v<f<p<u .
$$

Now we denote by $T^{*}$ the set consisting of elements $s, q, e, t, v, f, p$ and $u$. By Lemma 7 , we can verify that the elements of $T^{*}$ are multiplied together just as in the table (6) of the ordered semigroup $T_{1 L}$ in Example 1 in $\S 4$. Especially $T^{*}$ is a subsemigroup, which is clearly the subsemigroup generated by $(p, q)$. If $t \neq v$, then $T^{*}$ is isomorphic to $T_{1 L}$, while, if $t=v$, then $T^{*}$ is isomorphic to $T_{2 L}$.

Similarly, in the case when $p q \leqq q p$, we can show that, if $t \neq v$, then the subsemigroup $T^{*}$ generated by $(p, q)$ is isomorphic to $T_{1 R}$ and, if $t=v$, then $T^{*}$ is isomorphic to $T_{2 R}$.

THEOREM 3. Let $(p, q)$ be a regular pair of order 2 such that $q \leqq p$, and let $T^{*}$ be the subsemigroup of $S$ generated by $(p, q)$.

(a) If $q p \leqq p q$ and $q p^{2} q \neq p q^{2} p$, then $T^{*}$ is isomorphic to the $L$-typed ordered T-semigroup $T_{1 L}$;

(b) if $q p \leqq p q$ and $q p^{2} q=p q^{2} p$, then $T^{*}$ is isomorphic to the L-typed ordered T-semigroup $T_{2 L}$;

(c) if $p q \leqq q p$ and $q p^{2} q \neq p q^{2} q$, then $T^{*}$ is isomorphic to the $R$-typed ordered $T$-semigroup $T_{1 R}$;

(d) if $p q \leqq q p$ and $q p^{2} q=p q^{2} p$, then $T^{*}$ is isomorphic to the $R$-typed ordered $T$-semigroup $T_{2 R}$. 
6. Ordered $I$-semigroups. In this section, we give some examples of ordered semigroups each of which has a regular pair of infinite order.

EXAMPLE 1. The set of all integers forms an ordered semigroup with respect to the usual order and the usual addition. We denote this ordered semigroup by $I_{0}$. $I_{0}$ is even an ordered group.

EXAMPLE 2. Let $U$ be an ordered semigroup consisting of two elements -1 and 1 , with the usual order and the left singular multiplication:

$$
a b=a \text { for every } a, b \in U \text {. }
$$

We consider the lexicographically ordered direct product of $I_{0}$ and $U$, that is, the system $I_{1 L}$ consisting of pairs $(i, a)$ with $i \in I_{0}, a \in U$, in which the order and the multiplication are defined by

$$
\begin{aligned}
& (i, a)<(j, b) \quad \text { if } i<j \text { or } i=j, a<b ; \\
& (i, a)(j, b)=(i+j, a b)=(i+j, a) .
\end{aligned}
$$

It can easily be verified that this system $I_{1 L}$ is an ordered semigroup. (Here we remark that lexicographically ordered direct product of two ordered semigroups is not always an ordered semigroup.) In $I_{1 L}$, the subsemigroup, consisting of elements with the second component 1 , is isomorphic to the ordered semigroup $I_{0}$.

ExAMPle 3. Let $V$ be a system consisting of six elements with the order

$$
e_{2}<e_{1}<t<v<f_{1}<f_{2}
$$

and with the multiplication table:

\begin{tabular}{l|llllll} 
& $e_{2}$ & $e_{1}$ & $t$ & $v$ & $f_{1}$ & $f_{2}$ \\
\hline$e_{2}$ & $e_{2}$ & $e_{2}$ & $e_{2}$ & $e_{2}$ & $e_{2}$ & $e_{2}$ \\
$e_{1}$ & $e_{2}$ & $e_{1}$ & $t$ & $t$ & $t$ & $t$ \\
$t$ & $t$ & $t$ & $t$ & $t$ & $t$ & $t$ \\
$v$ & $v$ & $v$ & $v$ & $v$ & $v$ & $v$ \\
$f_{1}$ & $v$ & $v$ & $v$ & $v$ & $f_{1}$ & $f_{2}$ \\
$f_{2}$ & $f_{2}$ & $f_{2}$ & $f_{2}$ & $f_{2}$ & $f_{2}$ & $f_{2}$.
\end{tabular}

It can be verified that $V$ is an ordered idempotent semigroup. Now we define two mappings $\varphi$ and $\psi$ of $V$ into itself: 


$$
\begin{aligned}
& \varphi\left(e_{2}\right)=\varphi\left(e_{1}\right)=\varphi(t)=\varphi(v)=e_{2}, \quad \varphi\left(f_{1}\right)=e_{1}, \quad \varphi\left(f_{2}\right)=t ; \\
& \psi\left(e_{2}\right)=v, \quad \psi\left(e_{1}\right)=f_{1}, \quad \psi(t)=\psi(v)=\psi\left(f_{1}\right)=\psi\left(f_{2}\right)=f_{2} .
\end{aligned}
$$

As is easily seen, these mappings have the following properties:

(a) both $\varphi$ and $\psi$ are monotone:

$$
g \leqq h \text { implies } \varphi(g) \leqq \varphi(h), \psi(g) \leqq \psi(h) \text {; }
$$

(b) both $\varphi$ and $\psi$ are semigroup-homomorphisms:

$$
\varphi(g h)=\varphi(g) \varphi(h), \quad \psi(g h)=\psi(g) \psi(h) \text { for every } g, h \in V \text {; }
$$

(c) $\varphi(\varphi(g))=e_{2}, \psi(\psi(g))=f_{2}$ for every $g \in V$;

(d) $\varphi(g) e_{1}=e_{1} \varphi(g)=\varphi(g), \psi(g) f_{1}=f_{1} \psi(g)=\psi(g)$ for every $g \in V$;

(e) $\varphi(\psi(g))=e_{1} g, \psi(\varphi(g))=f_{1} g$ for every $g \in V$.

We consider the system $K$, consisting of pairs $(i, g)$ with $i \in I_{0}, g \in V$, in which the order is defined lexicographically and the multiplication is defined by

$$
(i, g)(j, h)= \begin{cases}\left(i+j, g e_{2}\right) & \text { if } i \leqq-2, \\ (i+j, g \varphi(h)) & \text { if } i=-1, \\ (i+j, g h) & \text { if } i=0, \\ (i+j, g \psi(h)) & \text { if } i=1, \\ \left(i+j, g f_{2}\right) & \text { if } i \geqq 2 .\end{cases}
$$

Using the properties (a)-(e) of $\varphi$ and $\psi$, we can prove that $K$ is an ordered semigroup. Finally we consider the subset $I_{2 L}$ of $K$, consisting of elements with

$$
\begin{aligned}
& i \leqq-2, g \neq e_{1}, f_{1}, \text { or } \\
& i=-1, g \neq f_{1}, \text { or } \\
& i=0, g \text { arbitrary, or } \\
& i=1, g \neq e_{1} \text { or } \\
& i \geqq 2, g \neq e_{1}, f_{1} .
\end{aligned}
$$

It can also be proved that $I_{2 L}$ is closed with respect to the multiplication, and so forms an ordered semigroup. In $I_{2 L}$, the subsemigroup consisting of elements with the second component $e_{2}$ or $f_{2}$, is isomorphic to $I_{1 L}$, and the subsemigroup consisting of elements with the second component $e_{2}$ is isomorphic to $I_{0}$.

ExAmple 4. Let $V, \varphi$ and $\psi$ be the same as in the preceding Example 3. We consider the system $K^{\prime}$, consisting of pairs $(i, g)$ with $i \in I_{0}, g \in V$, in which the order is defined lexicographically and the multiplication is defined by 


$$
(i, g)(j, h)= \begin{cases}\left(i+j, g f_{2}\right) & \text { if } i \leqq-2, \\ (i+j, g \psi(h)) & \text { if } i=-1 \\ (i+j, g h) & \text { if } i=0 \\ (i+j, g \varphi(h)) & \text { if } i=1 \\ \left(i+j, g e_{2}\right) & \text { if } i \geqq 2 .\end{cases}
$$

In a similar way as in Example 3, we can prove that $K^{\prime}$ is an ordered semigroup, in which the subset $I_{3 L}$, consisting of elements $(i, g)$ with

$$
\begin{aligned}
& i \leqq-2, g \neq e_{1}, f_{1}, \text { or } \\
& i=-1, g \neq e_{1}, \text { or } \\
& i=0, g \text { arbitrary, or } \\
& i=1, g \neq f_{1}, \text { or } \\
& i \geqq 2, g=e_{1}, f_{1},
\end{aligned}
$$

forms an ordered semigroup. In $I_{3 L}$, the subsemigroup consisting of elements with the second component $e_{2}$ or $f_{2}$, is isomorphic to $I_{1 L}$, and the subsemigroup consisting of elements with the second component $e_{2}$ is isomorphic to $I_{0}$.

EXAMPLE 5. We denote by $I_{4 L}$ the ordered semigroup constructed from $I_{2 L}$ by identifying $(i, t)$ and $(i, v)$ for every $i \in I_{0}$. It can be seen that this identification is possible.

EXAMPLE 6 . The ordered semigroup $I_{5 L}$ constructed from $I_{3 L}$ by identifying $(i, t)$ and $(i, v)$ for every $i \in I_{0}$.

EXAmple 7. The ordered semigroup $I_{1 R}$ which is dual to $I_{1 L}$.

EXAMPle 8. The ordered semigroup $I_{2 R}$ which is dual to $I_{2 L}$.

ExAMPle 9. The ordered semigroup $I_{3 R}$ which is dual to $I_{3 L}$.

EXAMPLE 10. The ordered semigroup $I_{4 R}$ which is dual to $I_{4 L}$.

EXAMPLE 11. The ordered semigroup $I_{5 R}$ which is dual to $I_{5 L}$.

These eleven ordered semigroups $I_{0}, I_{1 L}, I_{2 L}, \cdots, I_{5 R}$ are called ordered I-semigroups, in which $I_{0}$ is called the fundamental ordered $I$-semigroup. Every ordered $I$-semigroup contains a subsemigroup which is isomorphic to the fundamental ordered $I$-semigroup $I_{0}$.

7. Regular pair of infinite order (1). In this section, we characterize the subsemigroup generated by a regular pair of infinite order 
under some conditions. For brevity, in this section, always we denote by $(p, q)$ a regular pair of infinite order such that $q \leqq p$ and set

$$
\begin{aligned}
e_{n} & =\min \left\{q^{n} p^{n}, p^{n} q^{n}\right\}, \quad f_{n}=\max \left\{q^{n} p^{n}, p^{n} q^{n}\right\} \quad(n=1,2,3, \cdots), \\
t & =\min \left\{e_{1} f_{1}, f_{1} e_{1}\right\}, \quad v=\max \left\{e_{1} f_{1}, f_{1} e_{1}\right\} .
\end{aligned}
$$

LEMma 9. $\cdots<q^{3}<q^{2}<q<\cdots \leqq e_{3} \leqq e_{2} \leqq e_{1} \leqq t \leqq v \leqq f_{1} \leqq$ $f_{2} \leqq f_{3} \leqq \cdots<p<p^{2}<p^{3}<\cdots$.

Proof. By Lemma 2, $e_{1} \leqq t \leqq v \leqq f_{1}$. By Lemma 6, $q$ is negative and $p$ is positive, and so $q^{n+1}<q^{n}, p^{n}<p^{n+1}$ for every natural number $n$. First we suppose that $q p \leqq p q$. Then

$$
q^{n+1} p^{n+1}=q^{n}(q p) p^{n} \leqq q^{n}(p q) p^{n}=q^{n} p^{n},
$$

and similarly $p^{n+1} q^{n+1} \geqq p^{n} q^{n}$. Hence we obtain, for every natural number $n$,

$$
\begin{aligned}
e_{n+1}=q^{n+1} p^{n+1} & \leqq e_{n}=q^{n} p^{n} \leqq e_{1}=q p \leqq f_{1}=p q \\
& \leqq f_{n}=p^{n} q^{n} \leqq f_{n+1}=p^{n+1} q^{n+1}
\end{aligned}
$$

Finally, by Corollary 1 of Lemma 5,

$$
q q^{n}=q^{n+1}<q^{n}=q^{n} p^{n} q^{n}=e_{n} q^{n}, \quad f_{n} p^{n}=p^{n} q^{n} p^{n}=p^{n}<p^{n+1}=p p^{n}
$$

and so $q<e_{n}, f_{n}<p$. In the case when $p q \leqq q p$, we can prove this theorem in a similar way.

CoROLlaRY. For every natural number $n$, both $e_{n}$ and $f_{n}$ are idempotent. If $q p \leqq p q$, then $e_{n}=q^{n} p^{n}, f_{n}=p^{n} q^{n}$. If $p q \leqq q p$, then $e_{n}=p^{n} q^{n}, f_{n}=q^{n} p^{n}$.

LEMMA 10. For two natural numbers $m$ and $n$ such that $m<n$,

$$
e_{m} e_{n}=e_{n} e_{m}=e_{n}, \quad f_{m} f_{n}=f_{n} f_{m}=f_{n} .
$$

Proof. We prove only the first assertion in the case when $q p \leqq$ $p q$. By the preceding Corollary and Corollary 1 of Lemma 5,

$$
\begin{aligned}
& e_{m} e_{n}=q^{m} p^{m} q^{n} p^{n}=\left(q^{m} p^{m} q^{m}\right) q^{n-m} p^{n}=q^{m} q^{n-m} p^{n}=e_{n}, \\
& e_{n} e_{m}=q^{n} p^{n} q^{m} p^{m}=q^{n} p^{n-m}\left(p^{m} q^{m} p^{m}\right)=q^{n} p^{n-m} p^{m}=e_{n} .
\end{aligned}
$$

Now we remark that two relations

$$
e_{2} f_{1} \geqq e_{1}, \quad e_{2} f_{1}=e_{1} f_{1}
$$

are equivalent to each other. In fact, if $e_{2} f_{1} \geqq e_{1}$, then

$$
e_{2} f_{1}=\left(e_{2} f_{1}\right) f_{1} \geqq e_{1} f_{1} \geqq e_{2} f_{1},
$$


and so $e_{2} f_{1}=e_{1} f_{1}$. If $e_{2} f_{1}=e_{1} f_{1}$, then $e_{2} f_{1}=e_{1} f_{1} \geqq e_{1}^{2}=e_{1}$.

Similarly we can prove that each of the following sets of relations consists of equivalent relations:

$$
\begin{array}{ll}
f_{2} e_{1} \leqq f_{1}, & f_{2} e_{1}=f_{1} e_{1} ; \\
f_{1} e_{2} \geqq e_{1}, & f_{1} e_{2}=f_{1} e_{1}, \\
e_{1} f_{2} \leqq f_{1}, & e_{1} f_{2}=e_{1} f_{1} .
\end{array}
$$

Also the three relations

$$
f_{2} e_{1} \geqq f_{1}, \quad f_{2} e_{1}=f_{2}, \quad f_{2} e_{2}=f_{2}
$$

are equivalent to each other. In fact, if $f_{2} e_{1} \geqq f_{1}$, then

$$
f_{2} \geqq f_{2} e_{1}=f_{2}\left(f_{2} e_{1}\right) \geqq f_{2} f_{1}=f_{2},
$$

and so $f_{2} e_{1}=f_{2}$. If $f_{2} e_{1}=f_{2}$, then, without loss of generality by assuming that $q p \leqq p q$, we have $q^{2}=q^{2} f_{2}=q^{2} f_{2} e_{1}=q^{3} p$, and so $q^{2}=q^{4} p^{2}$. Therefore $f_{2}=p^{2} q^{2}=p^{2} q^{4} p^{2}=f_{2} e_{2}$. Finally, if $f_{2} e_{2}=f_{2}$, then $f_{2} e_{1} \geqq$ $f_{2} e_{2}=f_{2} \geqq f_{1}$.

Similarly we can prove that each of the following sets of relations consists of equivalent relations:

$$
\begin{array}{lll}
e_{2} f_{1} \leqq e_{1}, & e_{2} f_{1}=e_{2}, & e_{2} f_{2}=e_{2} ; \\
e_{1} f_{2} \geqq f_{1}, & e_{1} f_{2}=f_{2}, & e_{2} f_{2}=f_{2} ; \\
f_{1} e_{2} \leqq e_{1}, & f_{1} e_{2}=e_{2}, & f_{2} e_{2}=e_{2} .
\end{array}
$$

In what follows, we refer to the above-mentioned sets of equivalent relations as $(\mathrm{A}),(\mathrm{B}), \cdots,\left(\mathrm{D}^{\prime}\right)$, as shown at the left end of each line.

LEMmA 11. If either (C) or $\left(\mathrm{C}^{\prime}\right)$ holds, then $e_{2}=e_{3}=\ldots$. If either (D) or $\left(\mathrm{D}^{\prime}\right)$ holds, then $f_{2}=f_{3}=\cdots$.

Proof. We prove only that (C) implies $e_{2}=e_{3}=\ldots$ in the case when $q p \leqq p q$. In this case, as is shown in the proof of equivalence of relations in (C), we have $q^{2}=q^{3} p$, and so

$$
e_{2}=q^{2} p^{2}=e_{3}=q^{3} p^{3}=e_{4}=q^{4} p^{4}=\cdots \text {. }
$$

LEMMA 12. If $(p, q)$ is L-typed, then

(a) (C) is equivalent to $\left(\mathrm{A}^{\prime}\right)$, and $(\mathrm{D})$ is equivalent to $\left(\mathrm{B}^{\prime}\right)$;

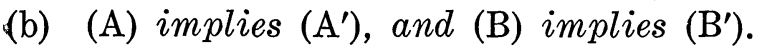

Proof. (a) We prove only that (C) is equivalent to $\left(\mathrm{A}^{\prime}\right)$ in the 
case when $q p \leqq p q$. If (C) holds, then, as is shown in the proof of equivalence of relations in (C), we have $q^{2}=q^{3} p$, and so

$$
f_{1} e_{2}=p q^{3} p^{2}=p q^{2} p=f_{1} e_{1} .
$$

Thus ( $\left.\mathrm{A}^{\prime}\right)$ holds. If $\left(\mathrm{A}^{\prime}\right)$ holds, then, by Lemma $3, f_{1} e_{1} f_{1}=f_{1} e_{1}$, and so

$$
\begin{aligned}
f_{2} & =p^{2} q^{2}=p f_{1} e_{1} q=p f_{1} e_{2} q=p^{2} q^{3} p^{2} q=f_{2} e_{1} f_{1} \\
& =\left(f_{2} f_{1}\right) e_{1} f_{1}=f_{2}\left(f_{1} e_{1}\right)=f_{2} e_{1} .
\end{aligned}
$$

Thus (C) holds. (b) We prove only (A) implies $\left(\mathrm{A}^{\prime}\right)$. By Lemma 3 and $(\mathrm{A})$,

$$
\begin{aligned}
& \left(f_{1} e_{1}\right)\left(f_{1} e_{2}\right)=\left(f_{1} e_{1} f_{1}\right) e_{2}=f_{1} e_{1} e_{2}=f_{1} e_{2}, \\
& \left(f_{1} e_{2}\right)\left(f_{1} e_{1}\right)=f_{1}\left(e_{2} f_{1}\right) e_{1}=f_{1} e_{1} f_{1} e_{1}=f_{1} e_{1} .
\end{aligned}
$$

Hence $f_{1} e_{1} \equiv f_{1} e_{2}(R)$. Therefore, by the assumption of being $L$-typed. of $(p, q)$, we obtain $f_{1} e_{1}=f_{1} e_{2}$.

Lemma 12'. If $(p, q)$ is R-typed, then

(a) $\left(\mathrm{C}^{\prime}\right)$ is equivalent to $(\mathrm{A})$, and $\left(\mathrm{D}^{\prime}\right)$ is equivalent to $(\mathrm{B})$;

(b) ( $\left.\mathrm{A}^{\prime}\right)$ implies (A), and $\left(\mathrm{B}^{\prime}\right)$ implies (B).

We divide the investigation of a regular pair of infinite order into two cases:

Case 1. the case when $e_{2} \equiv e_{1} f_{1}\left(D_{E}\right)$;

Case 2. the case when $e_{2} \not \equiv e_{1} f_{1}\left(D_{E}\right)$.

In the rest of this section we study Case 1, and Case 2 will be: studied in $\S 9$.

Case 1 is divided into two subcases:

Case 1L. the subcase of Case 1 when $(p, q)$ is L-typed;

Case $1 R$. the subcase of Case 1 when $(p, q)$ is R-typed.

Now we consider Case $1 L$, that is, suppose that $(p, q)$ is an $L$ typed regular pair of infinite order such that $q \leqq p$ and $e_{2} \equiv e_{1} f_{1}\left(D_{E}\right)$. Then $e_{2} \equiv e_{1} f_{1} \equiv f_{1} e_{1}(L)$, and so $e_{2}=e_{2}\left(e_{1} f_{1}\right)=e_{2} f_{1}, f_{1} e_{1}=\left(f_{1} e_{1}\right) e_{2}=f_{1} e_{2}$. Hence (D) and ( $\left.\mathrm{A}^{\prime}\right)$ hold. Then, by Lemma 12, also $\left(\mathrm{B}^{\prime}\right)$ and $(\mathrm{C})$ hold. Moreover, by Lemma 11, we have

$$
e_{2}=e_{3}=\cdots, \quad f_{2}=f_{3}=\cdots .
$$

Furthermore, by Lemma 3, 


$$
t=e_{1} f_{1}=e_{1} f_{1} e_{1}, \quad v=f_{1} e_{1}=f_{1} e_{1} f_{1} .
$$

We denote by $E$ the set consisting of $e_{2}, e_{1}, t, v, f_{1}$ and $f_{2}$. Then, by $\left(\mathrm{A}^{\prime}\right),\left(\mathrm{B}^{\prime}\right),(\mathrm{C}),(\mathrm{D}),(13)$ and (14), we can verify that elements of $E$ are multiplied together just as in the table (9) in Example 3 in $\S 6$.

We divide Case $1 L$ into two subcases:

Case 1L1. the subcase of Case $1 L$ when $q p \leqq p q$;

Case 1L2. the subcase of Case $1 L$ when $p q \leqq q p$.

First we consider Case $1 L 1$. In this case, by Corollary of Lemma 9 , we have $e_{n}=q^{n} p^{n}$ and $f_{n}=p^{n} q^{n}$. Therefore, by $f_{2} e_{1}=f_{2}$ and $e_{2} f_{1}=$ $e_{2}$, we obtain

$$
p^{2}=p^{3} q, \quad q^{2}=q^{3} p,
$$

and, by $e_{1} f_{1}=e_{1} f_{1} e_{1}$ and $f_{1} e_{1}=f_{1} e_{1} f_{1}$,

$$
p^{2} q=p^{2} q^{2} p, \quad q^{2} p=q^{2} p^{2} q .
$$

Now we consider the mappings $\varphi$ and $\psi$ of $E$ into itself which have been defined in Example 3 in $\S 6$, that is,

$$
\begin{aligned}
& \varphi\left(e_{2}\right)=\varphi\left(e_{1}\right)=\varphi(t)=\varphi(v)=e_{2}, \quad \varphi\left(f_{1}\right)=e_{1}, \quad \varphi\left(f_{2}\right)=t ; \\
& \psi\left(e_{2}\right)=v, \quad \psi\left(e_{1}\right)=f_{1}, \quad \psi(t)=\psi(v)=\psi\left(f_{1}\right)=\psi\left(f_{2}\right)=f_{2} .
\end{aligned}
$$

Then, by (15) and (16), it is easily verified that

$$
q g=\varphi(g) q, \quad p g=\psi(g) p \text { for every } g \in E .
$$

Especially we have $q e_{2}=e_{2} q, p f_{2}=f_{2} p$, and so

$$
q^{n} e_{2}=e_{2} q^{n}, \quad p^{n} f_{2}=f_{2} p^{n} \text { for every natural number } n \text {. }
$$

Moreover by (17)

$$
\begin{aligned}
& q^{2} g=q \varphi(g) q=\varphi(\varphi(g)) q^{2}=e_{2} q^{2}=q^{2}, \\
& p^{2} g=p \psi(g) p=\psi(\psi(g)) p^{2}=f_{2} p^{2}=p^{2},
\end{aligned}
$$

and so

$$
q^{n} g=e_{2} q^{n}=q^{n}, \quad p^{n} g=f_{2} p^{n}=p^{n} \text { for every } g \in E, n \geqq 2 .
$$

By (18) $e_{2} q^{m+1} p^{n+1}=e_{2} q^{m} e_{1} p^{n}=q^{m} e_{2} e_{1} p^{n}=q^{m} e_{2} p^{n}=e_{2} q^{m} p^{n}$ and $f_{2} p^{m+1} q^{n+1}=$ $f_{2} p^{m} q^{n}$ in a similar way. Hence

$$
e_{2} q^{m} p^{n}= \begin{cases}e_{2} q^{m-n} & \text { if } m>n, \\ e_{2} & \text { if } m=n, \\ e_{2} p^{n-m} & \text { if } m<n,\end{cases}
$$




$$
f_{2} p^{m} q^{n}= \begin{cases}f_{2} p^{m-n} & \text { if } m>n, \\ f_{2} & \text { if } m=n, \\ f_{2} q^{n-m} & \text { if } m<n .\end{cases}
$$

By (19) and (20),

$$
\text { if } m \geqq 2,\left\{\begin{array}{l}
g q^{m} h q^{n}=g e_{2} q^{m+n}, \\
g q^{m} h=g e_{2} q^{m}, \\
g q^{m} h p^{n}= \begin{cases}g e_{2} q^{m-n} & \text { when } m>n, \\
g e_{2} & \text { when } m=n, \\
g e_{2} p^{n-m} & \text { when } m<n .\end{cases}
\end{array}\right.
$$

Similarly by (19) and (21),

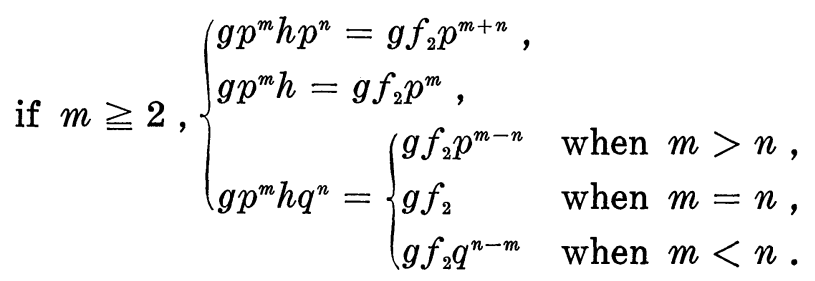

We have mentioned in $\S 6$ that $\varphi(g) e_{1}=\varphi(g)$ and $\psi(g) f_{1}=\psi(g)$. Hence by (17),

$$
\begin{aligned}
& \left\{\begin{array}{l}
g q h q^{n}=g \varphi(h) q^{n+1}, \\
g q h=g \varphi(h) q, \\
g q h p=g \varphi(h), \\
g q h p^{n}=g \varphi(h) p^{n-1}
\end{array} \text { if } n \geqq 2 ;\right. \\
& \left\{\begin{array}{l}
g p h p^{n}=g \psi(h) p^{n+1}, \\
g p h=g \psi(h) p, \\
g p h q=g \psi(h), \\
g p h q^{n}=g \psi(h) q^{n-1}
\end{array} \text { if } n \geqq 2 .\right.
\end{aligned}
$$

We denote by $I^{*}$ the set consisting of elements of the forms $g q^{n}$ or $g p^{n}$ or $g$ with $g \in E$ and natural number $n$. By (22)-(25), we see that $I^{*}$ is a subsemigroup, which is then clearly the subsemigroup generated by the regular pair $(p, q)$.

Since $q=e_{1} q$ and $p=f_{1} p$, we have

$$
v q=f_{1} q, \quad e_{1} p=t p .
$$

By (26) and relations $e_{2} q^{2}=q^{2}=e_{1} q^{2}, f_{2} p^{2}=p^{2}=f_{1} p^{2}$,

$$
e_{2} q^{n}=e_{1} q^{n}, \quad v q^{n}=f_{1} q^{n}, \quad e_{1} p^{n}=t p^{n}, \quad f_{1} p^{n}=f_{2} p^{n} \quad \text { for } n \geqq 2 .
$$


By (18), $q^{2}\left(f_{2} q^{n+1}\right)=q^{n+3}<q^{n+2}=e_{2} q^{n+2}=q^{2}\left(e_{2} q^{n}\right)$ and $q^{2}\left(f_{2} q\right)=q^{3}<q^{2}=$ $e_{2} q^{2}=q^{2} e_{2}$, and so

$$
f_{2} q^{n+1}<e_{2} q^{n}, \quad f_{2} q<e_{2} .
$$

Similarly

$$
f_{2}<e_{2} p, \quad f_{2} p^{n}<e_{2} p^{n+1} .
$$

Thus the elements of $I^{*}$ are ordered by

$$
\begin{aligned}
\cdots & <e_{2} q^{2} \leqq t q^{2} \leqq v q^{2} \leqq f_{2} q^{2}<e_{2} q \leqq e_{1} q \leqq t q \leqq v q \leqq f_{2} q \\
& <e_{2} \leqq e_{1} \leqq t \leqq v \leqq f_{1} \leqq f_{2}<e_{2} p \leqq t p \leqq v p \leqq f_{1} p \leqq f_{2} p \\
& <e_{2} p^{2} \leqq t p^{2} \leqq v p^{2} \leqq f_{2} p^{2}<\cdots .
\end{aligned}
$$

LEMma 13. In this Case $1 L 1$, all the following relations are equivalent to each other:

$$
\begin{gathered}
\cdots, e_{2} q^{2}=t q^{2}, v q^{2}=f_{2} q^{2}, e_{2} q=e_{1} q, e_{1} q=t q, v q=f_{2} q, \\
e_{2}=e_{1}, e_{1}=t, v=f_{1}, f_{1}=f_{2}, e_{2} p=t p, v p=f_{1} p, \\
f_{1} p=f_{2} p, e_{2} p^{2}=t p^{2}, v p^{2}=f_{2} p^{2}, \cdots .
\end{gathered}
$$

Proof. First we prove that the four relations $e_{2}=e_{1}, e_{1}=t, v=$ $f_{1}, f_{1}=f_{2}$ are equivalent to each other. In fact, if $e_{2}=e_{1}$, then

$$
e_{1}=e_{2}=e_{2} f_{1}=e_{1} f_{1}=t \text {. }
$$

If $e_{1}=t$, then

$$
f_{1}=p e_{1} q=p t q=f_{2} .
$$

It can similarly be proved that $f_{1}=f_{2}$ implies $v=f_{1}$ and that $v=f_{1}$ implies $e_{2}=e_{1}$. Next we prove that $v q^{n}=f_{2} q^{n}$ are equivalent to $v=$ $f_{1}$. In fact, if $v q^{n}=f_{2} q^{n}$, then, by taking account of the table (9), $v=v q^{n} p^{n}=f_{2} q^{n} p^{n}=f_{2}$, and so $v=f_{1}$. If $v=f_{1}$, then, by the result proved above, $v=f_{1}=f_{2}$, and so $v q^{n}=f_{2} q^{n}$. Similarly we can prove that each of the remaining relations is equivalent to one of the relations $e_{2}=e_{1}, e_{1}=t, v=f_{1}, f_{1}=f_{2}$.

In a similar way, we can prove the following

Lemma 14. In this Case $1 L 1$, all the following relations are equivalent to each other:

$$
\cdots, t q^{2}=v q^{2}, t q=v q, t=v, t p=v p, t p^{2}=v p^{2}, \cdots .
$$

Now we study Case $1 L 1$ by dividing into subcases.

$1^{\circ}$. Subcase of Case $1 L 1$ when $e_{2} \neq e_{1}, t \neq v$. 
In this subcase, by Lemmas 13 and 14 , all the elements of $I^{*}$ written in (30) are different from each other. We consider a mapping of $I_{2 L}$ of Example 3 in $\S 6$ into $I^{*}$ :

$$
(i, g) \rightarrow \begin{cases}g p^{i} & \text { if } i>0 \\ g & \text { if } i=0 \\ g q^{-i} & \text { if } i<0\end{cases}
$$

By (11) in $\S 6$, this mapping is one-to-one and onto. Moreover, it is order-preserving. Furthermore, comparing (10) in $\S 6$ and (22)-(25), we see that this mapping is an isomorphism. Thus $I^{*}$ is isomorphic to $I_{2 L}$. We remark, by the above isomorphism, $\left(-1, e_{1}\right)$ and $\left(1, f_{1}\right)$ are mapped into $q$ and $p$, respectively.

$2^{\circ}$. Subcase of Case $1 L 1$ when $e_{2} \neq e_{1}, t=v$.

In this subcase, by Lemmas 13 and 14 and the consideration of $1^{\circ}, I^{*}$ is isomorphic to $I_{4 L}$.

$3^{\circ}$. Subcase of Case $1 L 1$ when $e_{2}=e_{1}, t \neq v$.

By Lemmas 13 and 14, $I^{*}$ consists of elements

$$
\begin{aligned}
\cdots<e_{2} q^{2}=q^{2} & <f_{2} q^{2}<e_{2} q=q<f_{2} q<e_{2} \\
& <f_{2}<e_{2} p<f_{2} p=p<e_{2} p^{2}<f_{2} p^{2}=p^{2}<\cdots,
\end{aligned}
$$

and, by (22)-(25), we have

$$
\begin{aligned}
& \left\{\begin{array}{l}
g q^{m} h q^{n}=g q^{m+n}, \\
g q^{m} h=g q^{m}, \\
g q^{m} h p^{n}= \begin{cases}g q^{m-n} & \text { if } m>n, \\
g & \text { if } m=n, \\
g p^{n-m} & \text { if } m<n,\end{cases}
\end{array}\right. \\
& \left\{\begin{array}{l}
g p^{m} h p^{n}=g p^{m+n}, \\
g p^{m} h=g p^{m}, \\
g p^{m} h q^{n}= \begin{cases}g p^{m-n} & \text { if } m>n, \\
g & \text { if } m=n, \\
g q^{n-m} & \text { if } m<n .\end{cases}
\end{array}\right.
\end{aligned}
$$

Also we have

$$
g h q^{n}=g q^{n}, \quad g h=g, \quad g h p^{n}=g p^{n} .
$$

Thus, in this subcase, the mapping 
(33)

$$
\begin{array}{r}
(i,-1) \rightarrow \begin{cases}e_{2} p^{i} & \text { if } i>0, \\
e_{2} & \text { if } i=0, \\
e_{2} q^{-i} & \text { if } i<0,\end{cases} \\
(i, 1) \rightarrow \begin{cases}f_{2} p^{i} & \text { if } i>0, \\
f_{2} & \text { if } i=0, \\
f_{2} q^{-i} & \text { if } i<0,\end{cases}
\end{array}
$$

is an isomorphism of $I_{1 L}$ onto $I^{*}$. We rernark, in this isomorphism, $(-1,-1)$ and $(1,1)$ are mapped into $q$ and $p$, respectively.

$4^{\circ}$. Subcase of Case $1 L 1$ when $e_{2}=e_{1}, t=v$.

In this subcase, by Lemmas 13 and $14, I^{*}$ consists of elements

$$
\cdots<q^{3}<q^{2}<q<e_{2}=e_{1}=t=v=f_{1}=f_{2}<p<p^{2}<p^{3}<\cdots,
$$

and, by (22)-(25),

$$
\begin{gathered}
q^{m} p^{n}=p^{n} q^{m}= \begin{cases}q^{m-n} & \text { if } m>n, \\
e_{1} & \text { if } m=n, \\
p^{n-m} & \text { if } m<n,\end{cases} \\
e_{1} q^{m}=q^{m} e_{1}=q^{m}, \quad e_{1} p^{m}=p^{m} e_{1}=p^{m} .
\end{gathered}
$$

Thus the mapping

$$
i \rightarrow \begin{cases}p^{i} & \text { if } i>0, \\ e_{1} & \text { if } i=0, \\ q^{-i} & \text { if } i<0\end{cases}
$$

is an isomorphism of $I_{0}$ onto $I^{*}$.

Next we consider Case $1 L 2$.

$1^{\circ}$. Subcase of Case 1 L2 when $e_{2} \neq e_{1}, t \neq v$.

We can prove, in a similar way as in the corresponding subcase of Case $1 L 1$, that the subsemigroup $I^{*}$ generated by $(p, q)$ consists of elements

$$
\begin{aligned}
\cdots & <e_{2} q^{2}<t q^{2}<v q^{2}<f_{2} q^{2}<e_{2} q<t q<v q<f_{1} q=q<f_{2} q<e_{2} \\
& <e_{1}<t<v<f_{1}<f_{2}<e_{2} p<e_{1} p=p<t p<v p<f_{2} p<e_{2} p^{2} \\
& <t p^{2}<v p^{2}<f_{2} p^{2}<\cdots,
\end{aligned}
$$

and that the mapping given by the same formula (31) as in Case $1 L 1$ is an isomorphism of $I_{3 L}$ onto $I^{*}$. In particular, $\left(-1, f_{1}\right)$ and $\left(1, e_{1}\right)$ are mapped into $q$ and $p$, respectively. 
$2^{\circ}$. Subcase of Case 1 L2 when $e_{2} \neq e_{1}, t=v$.

The subsemigroup $I^{*}$ generated by $(p, q)$ is isomorphic to $I_{5 L}$.

$3^{\circ}$. Subcase of Case 1 L2 when $e_{2}=e_{1}, t \neq v$.

The subsemigroup $I^{*}$ generated by $(p, q)$ consists of elements

$$
\begin{aligned}
\cdots<e_{2} q^{2} & <f_{2} q^{2}=q^{2}<e_{2} q<f_{2} q=q<e_{2} \\
& <f_{2}<e_{2} p=p<f_{2} p<e_{2} p^{2}=p^{2}<f_{2} p^{2}<\cdots,
\end{aligned}
$$

and so consists of the same elements as in the corresponding subcase in Case 1L1. Also the multiplication in $I^{*}$ is given by the same formula (32)-(32") as in Case 1L1. Hence the mapping (33) is an isomorphism of $I_{1 L}$ onto $I^{*}$. However, in this subcase, $(-1,1)$ and $(1,-1)$ are mapped into $q$ and $p$, respectively.

$4^{\circ}$. Subcase of Case 1 L2 when $e_{2}=e_{1}, t=v$.

The subsemigroup $I^{*}$ generated by $(p, q)$ is the same, in all respects, as that in the corresponding subcase in Case $1 L 1$.

In Case $1 R$, we can argue in a similar way.

THEOREM 4. Using the notations given in (12), let $(p, q)$ be a regular pair of infinite order such that $q \leqq p$ and $e_{2} \equiv e_{1} f_{1}\left(D_{E}\right)$, let $I^{*}$ be the subsemigroup generated by $(p, q)$, and let $I_{0}-I_{5 R}$ be the ordered I-semigroups given in $\S 6$.

(a) If $e_{2}=e_{1}$ and $t=v$, then $I^{*}$ is isomorphic to $I_{0}$;

(b) if $(p, q)$ is L-typed, $e_{2}=e_{1}$ and $t \neq v$, then $I^{*}$ is isomorphic to $I_{1 L}$;

(c) if $(p, q)$ is L-typed, $q p \leqq p q, e_{2} \neq e_{1}$ and $t \neq v$, then $I^{*}$ is isomorphic to $I_{2 L}$;

(d) if $(p, q)$ is L-typed, $p q \leqq q p, e_{2} \neq e_{1}$ and $t \neq v$, then $I^{*}$ is isomorphic to $L_{3 L}$;

(e) if $(p, q)$ is L-typed, $q p \leqq p q, e_{2} \neq e_{1}$ and $t=v$, then $I^{*}$ is isomorphic to $I_{41}$;

(f) if $(p, q)$ is L-typed, $p q \leqq q p, e_{2} \neq e_{1}$ and $t=v$, then $I^{*}$ is isomorphic to $I_{5 L}$; to $I_{1 R}$;

(g) if $(p, q)$ is $R$-typed, $e_{2}=e_{1}$ and $t \neq v$, then $I^{*}$ is isomorphic

(h) if $(p, q)$ is $R$-typed, $p q \leqq q p, e_{2} \neq e_{1}$ and $t \neq v$, then $I^{*}$ is isomorphic to $I_{2 R}$;

(i) if $(p, q)$ is $R$-typed, $q p \leqq p q, e_{2} \neq e_{1}$ and $t \neq v$, then $I^{*}$ is isomorphic to $I_{3 R}$; 
(j) if $(p, q)$ is $R$-typed, $p q \leqq q p, e_{2} \neq e_{1}$ and $t=v$, then $I^{*}$ is isomorphic to $I_{4 R}$;

(k) if $(p, q)$ is R-typed, $q p \leqq p q, e_{2} \neq e_{1}$ and $t=v$, then $I^{*}$ is isomorphic to $I_{5 R}$.

COROLLARY. Under the assumptions of Theorem 4, I* contains subsemigroup isomorphic to the ordered additive group $I_{0}$ of integers.

8. Ordered $J$-semigroups. In $\S 6$, we gave examples of ordered semigroups each of which has a regular pair of infinite order. In this section, we give examples of another kind of such semigroups.

EXAMPLE 1. Let $J$ be the set of pairs $(m, n)$ of nonnegative integers with the multiplication

$$
(k, l)(m, n)= \begin{cases}(k+m-l, n) & \text { if } l \leqq m \\ (k, n+l-m) & \text { if } m \leqq l .\end{cases}
$$

As is well known, $J$ is an abstract semigroup (Lyapin [4] or Saitô [9]). It can be verified that the semigroup $J$ turns out to be an ordered semigroup when we define the order in $J$ by

$$
(k, l)<(m, n) \text { if } k+n<m+l \quad \text { or } k+n=m+l, k<m .
$$

This ordered semigroup is denoted by $J_{01}$.

Example 2. It can be verified that the semigroup $J$ in Example 1 turns out to be an ordered semigroup when we define the order in $J$ by

$$
(k, l)<(m, n) \text { if } k+n>m+l \quad \text { or } k+n=m+l, k<m .
$$

This ordered semigroup is denoted by $J_{02}$.

EXAMPLE 3. The ordered semigroup $J_{03}$, which is the semigroup $J$ with the order

$$
(k, l)<(m, n) \text { if } k+n<m+l \quad \text { or } k+n=m+l, k>m .
$$

ExAMPLE 4. The ordered semigroup $J_{04}$, which is the semigroup $J$ with the order

$$
(k, l)<(m, n) \quad \text { if } k+n>m+l \quad \text { or } k+n=m+l, k>m .
$$

In each of the ordered semigroups $J_{01}-J_{04},((0,1),(1,0))$ is a regular pair of infinite order, which generates the corresponding ordered semigroup. In $J_{01}$ and $J_{03},(0,1)$ is negative and $(1,0)$ is positive, while in $J_{02}$ and $J_{04},(0,1)$ is positive and $(1,0)$ is negative. 
EXAMPLE 5. Let $W$ be a system consisting of infinite elements ordered by

$$
e_{2}<e_{1}<t<v<f_{1}<f_{2}<\cdots,
$$

and with the following multiplication table:

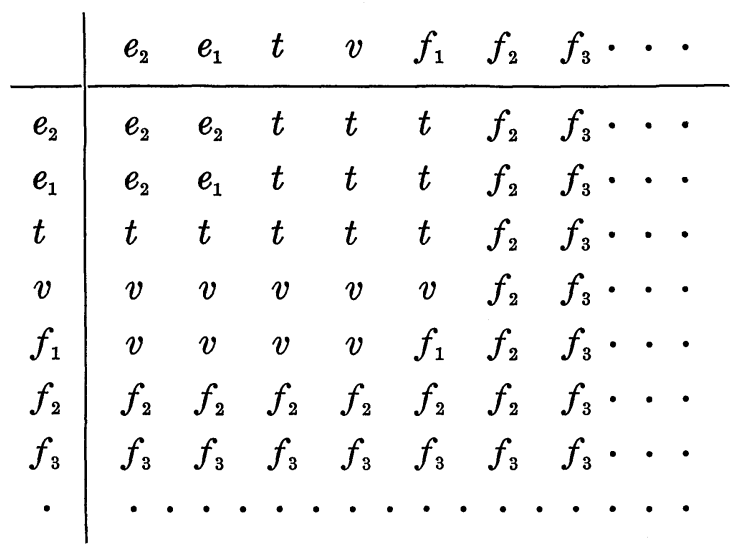

It can be verified that $W$ is an ordered idempotent semigroup. We define two mappings $\varphi$ and $\psi$ of $W$ into itself by

$$
\begin{gathered}
\varphi\left(e_{2}\right)=\varphi\left(e_{1}\right)=\varphi(t)=\varphi(v)=e_{2}, \varphi\left(f_{1}\right)=e_{1}, \\
\varphi\left(f_{2}\right)=t, \varphi\left(f_{3}\right)=f_{2}, \cdots ; \\
\psi\left(e_{2}\right)=v, \psi\left(e_{1}\right)=f_{1}, \psi(t)=\psi(v)=\psi\left(f_{1}\right)=f_{2}, \\
\psi\left(f_{2}\right)=f_{3}, \psi\left(f_{3}\right)=f_{4}, \cdots .
\end{gathered}
$$

As is easily seen, these mappings have the following properties:

(a) both $\varphi$ and $\psi$ are monotone;

(b) both $\varphi$ and \& are semigroup-homomorphisms;

(c) $\varphi(g) e_{1}=e_{1} \varphi(g)=\varphi(g), \psi(g) f_{1}=f_{1} \psi(g)=\psi(g)$ for every $g \in W$;

(d) $\varphi(\psi(g))=e_{1} g, \psi(\varphi(g))=f_{1} g$ for every $g \in W$.

For brevity, we use notations:

$$
\begin{aligned}
& \varphi^{1}(g)=\varphi(g), \varphi^{2}(g)=\varphi(\varphi(g)), \varphi^{3}(g)=\varphi(\varphi(\varphi(g))), \cdots ; \\
& \psi^{1}(g)=\psi(g), \psi^{2}(g)=\psi(\psi(g)), \psi^{3}(g)=\psi(\psi(\psi(g))), \cdots .
\end{aligned}
$$

Now we consider the system $H$, consisting of pairs $(i, g)$ with $i \in I_{0}$ and $g \in W$, where $I_{0}$ is the ordered additive group of integers as is defined in $\S 6$. In $H$, we define the order lexicographically and define the multiplication by

$$
(i, g)(j, h)= \begin{cases}\left(i+j, g \varphi^{-i}(h)\right) & \text { if } i<0 \\ (i+j, g h) & \text { if } i=0 \\ \left(i+j, g \psi^{i}(h)\right) & \text { if } i>0\end{cases}
$$


Using the properties (a)-(d) of $\varphi$ and $\psi$, we can prove that $H$ is an ordered semigroup. Finally, we consider the subset $J_{11 L}$ of $H$ consisting of elements with

$$
\begin{aligned}
& i \leqq-2, g \neq e_{1}, f_{1}, \text { or } \\
& i=-1, g \neq f_{1}, \text { or } \\
& i=0, g \text { arbitrary, or } \\
& i=1, g \geqq t, \text { or } \\
& i \geqq 2, g \geqq f_{i} .
\end{aligned}
$$

It can be verified that $J_{11 L}$ is closed with respect to the multiplication, and so forms an ordered semigroup. Here we remark that the ordered semigroup $J_{11 L}$ contains a subsemigroup which is isomorphic to $J_{01}$. In fact, we can verify that the following mapping of $J_{01}$ into $J_{11 L}$ is an isomorphism into $J_{11 L}$ :

$$
(m, n) \rightarrow \begin{cases}\left(m-n, e_{2}\right) & \text { if } m=0, \\ (m-n, t) & \text { if } m=1, \\ \left(m-n, f_{m}\right) & \text { if } m \geqq 2 .\end{cases}
$$

EXAMPLE 6. Let $W, \varphi$ and $\psi$ be the same as in the preceding Example 5. We can verify that the system $J_{12 L}$, consisting of pairs $(i, g)$ with $i \in I_{0}, g \in W$ which satisfies

$$
\begin{aligned}
& i \leqq-2, g \geqq f_{i}, \text { or } \\
& i=-1, g \geqq t, \text { or } \\
& i=0, g \text { arbitrary, or } \\
& i=1, g \neq f_{1}, \text { or } \\
& i \geqq 2, g \neq e_{1}, f_{1},
\end{aligned}
$$

forms an ordered semigroup, when we define the order lexicographically and define the multiplication by

$$
(i, g)(j, h)= \begin{cases}\left(i+j, g \psi^{-i}(h)\right) & \text { if } i<0 \\ (i+j, g h) & \text { if } i=0 \\ \left(i+j, g \varphi^{i}(h)\right) & \text { if } i>0\end{cases}
$$

Moreover we can verify that the mapping of $J_{02}$ into $J_{12 L}$ defined by

$$
(m, n) \rightarrow \begin{cases}\left(n-m, e_{2}\right) & \text { if } m=0, \\ (n-m, t) & \text { if } m=1, \\ \left(n-m, f_{m}\right) & \text { if } m \geqq 2\end{cases}
$$

is an isomorphism into $J_{12 L}$, and so $J_{12 L}$ contains a subsemigroup which is isomorphic to $J_{02}$. 
EXAMPLE 7. Let $W^{\prime}$ be the system consisting of infinite elements ordered by

$$
\cdots<e_{3}<e_{2}<e_{1}<t<v<f_{1}<f_{2},
$$

and with the multiplication table arising from the table (34) by means of replacing $e_{2}, e_{1}, t, v, f_{1}, f_{2}, f_{3}, \cdots$ by $f_{2}, f_{1}, v, t, e_{1}, e_{2}, e_{3}, \cdots$ respectively. It can be verified that $W^{\prime}$ is an ordered idempotent semigroup. We define two mappings $\chi$ and $\omega$ of $W^{\prime}$ into itself by

$$
\begin{gathered}
\chi\left(f_{2}\right)=\chi\left(f_{1}\right)=\chi(v)=\chi(t)=f_{2}, \chi\left(e_{1}\right)=f_{1}, \\
\chi\left(e_{2}\right)=v, \chi\left(e_{3}\right)=e_{2}, \cdots ; \\
\omega\left(f_{2}\right)=t, \omega\left(f_{1}\right)=e_{1}, \omega(v)=\omega(t)=\omega\left(e_{1}\right)=e_{2}, \\
\\
\omega\left(e_{2}\right)=e_{3}, \omega\left(e_{3}\right)=e_{4}, \cdots .
\end{gathered}
$$

In a similar way as in Example 5, the system $J_{13 L}$, consisting of pairs $(i, g)$ with $i \in I_{0}, g \in W^{\prime}$ which satisfies

$$
\begin{aligned}
& i \leqq-2, g \neq e_{1}, f_{1}, \text { or } \\
& i=-1, g \neq e_{1}, \text { or } \\
& i=0, g \text { arbitrary, or } \\
& i=1, g \leqq v, \text { or } \\
& i \geqq 2, g \leqq e_{i}
\end{aligned}
$$

forms an ordered semigroup, when we order it lexicographically and define the multiplication by

$$
(i, g)(j, h)= \begin{cases}\left(i+j, g \chi^{-i}(h)\right) & \text { if } i<0 \\ (i+j, g h) & \text { if } i=0 \\ \left(i+j, g \omega^{i}(h)\right) & \text { if } i>0\end{cases}
$$

The mapping of $J_{03}$ into $J_{13 L}$ defined by

$$
(m, n) \rightarrow \begin{cases}\left(m-n, f_{2}\right) & \text { if } m=0, \\ (m-n, v) & \text { if } m=1, \\ \left(m-n, e_{m}\right) & \text { if } m \geqq 2\end{cases}
$$

is an isomorphism into $J_{13 L}$, and so $J_{13 L}$ contains a subsemigroup which is isomorphic to $J_{03}$.

EXAMPLE 8. The ordered semigroup $J_{14 L}$ consists of pairs $(i, g)$ with $i \in I_{0}, g \in W^{\prime}$ which satisfies

$$
\begin{aligned}
& i \leqq-2, g \leqq e_{i}, \text { or } \\
& i=-1, g \leqq v \text {, or } \\
& i=0, g \text { arbitrary, or }
\end{aligned}
$$




$$
\begin{aligned}
& i=1, g \neq e_{1}, \text { or } \\
& i \geqq 2, g \neq e_{1}, f_{1} .
\end{aligned}
$$

It is ordered lexicographically and has the multiplication

$$
(i, g)(j, h)= \begin{cases}\left(i+j, g \omega^{-i}(h)\right) & \text { if } i<0, \\ (i+j, g h) & \text { if } i=0, \\ \left(i+j, g \chi^{i}(h)\right) & \text { if } i>0 .\end{cases}
$$

The mapping of $J_{04}$ into $J_{14 L}$ defined by

$$
(m, n) \rightarrow \begin{cases}\left(n-m, f_{2}\right) & \text { if } m=0, \\ (n-m, v) & \text { if } m=1, \\ \left(n-m, e_{m}\right) & \text { if } m \geqq 2\end{cases}
$$

is an isomorphism into $J_{14 L}$.

EXAMPLE 9. The ordered semigroup $J_{21 L}$ is constructed from $J_{11 L}$ by identifying elements contained in each of the following four pairs:

$$
\left(-1, e_{2}\right),\left(-1, e_{1}\right) ;\left(0, e_{2}\right),\left(0, e_{1}\right) ;(0, v),\left(0, f_{1}\right) ;(1, v),\left(1, f_{1}\right) \text {. }
$$

$J_{21 L}$ contains a subsemigroup which is isomorphic to $J_{01}$.

EXAMPLE 10. The ordered semigroup $J_{22 L}$ is constructed from $J_{12 L}$ by identifying elements contained in each of the following four pairs:

$$
(-1, v),\left(-1, f_{1}\right) ;\left(0, e_{2}\right),\left(0, e_{1}\right) ;(0, v),\left(0, f_{1}\right) ;\left(1, e_{2}\right),\left(1, e_{1}\right) \text {. }
$$

$J_{22 L}$ contains a subsemigroup which is isomorphic to $J_{02}$.

EXAMPLE 11. The ordered semigroup $J_{23 L}$ is constructed from $J_{13 L}$ by identifying elements contained in each of the following four pairs:

$$
\left(-1, f_{1}\right),\left(-1, f_{2}\right) ;\left(0, e_{1}\right),(0, t) ;\left(0, f_{1}\right),\left(0, f_{2}\right) ;\left(1, e_{1}\right),(1, t) \text {. }
$$

$J_{23 L}$ contains a subsemigroup which is isomorphic to $J_{03}$.

EXAMPLE 12. The ordered semigroup $J_{24 L}$ is constructed from $J_{14 L}$ by identifying elements contained in each of the following four pairs:

$$
\left(-1, e_{1}\right),(-1, t) ;\left(0, e_{1}\right),(0, t) ;\left(0, f_{1}\right),\left(0, f_{2}\right) ;\left(1, f_{1}\right),\left(1, f_{2}\right) \text {. }
$$

$J_{24 L}$ contains a subsemigroup which is isomorphic to $J_{04}$.

ExAMPLE 13. The ordered semigroup $J_{31}$ is constructed from $J_{11 L}$ by identifying $(i, t)$ and $(i, v)$ for each $i \leqq 1$. $J_{31}$ contains a subsemigroup which is isomorphic to $J_{01}$. 
EXAMPLE 14. The ordered semigroup $J_{32}$ is constructed from $J_{12 L}$ by identifying $(i, t)$ and $(i, v)$ for each $i \geqq-1$. $J_{32}$ contains a subsemigroup which is isomorphic to $J_{02}$. Here we remark that the ordered semigroup $J_{32}$ is isomorphic to the dual of ordered semigroup $J_{31}$. In fact, we can verify that the mapping of the dual of ordered semigroup $J_{31}$ into $J_{32}$ defined by

$$
(i, g) \rightarrow \begin{cases}\left(i, \psi^{-i}(g)\right) & \text { if } i<0 \\ (i, g) & \text { if } i=0 \\ \left(i, \varphi^{i}(g)\right) & \text { if } i>0\end{cases}
$$

is an isomorphism onto $J_{32}$.

EXAMPLE 15. The ordered semigroup $J_{33}$ is constructed from $J_{13 L}$ by identifying $(i, t)$ and $(i, v)$ for each $i \leqq 1$. $J_{33}$ contains a subsemigroup which is isomorphic to $J_{03}$.

EXAMPLE 16. The ordered semigroup $J_{34}$ is constructed from $J_{14 L}$ by identifying $(i, t)$ and $(i, v)$ for each $i \geqq-1$. $J_{34}$ contains a subsemigroup which is isomorphic to $J_{04}$. We remark that $J_{34}$ is isomorphic to the dual of ordered semigroup $J_{33}$.

EXAMPLES 17-24. The ordered semigroups $J_{11 R}, \cdots, J_{24 R}$ are multiplicative dual to $J_{11 L}, \cdots, J_{24 L}$, respectively.

These 24 ordered semigroups given above are called ordered $J$ semigroups. Ordered $J$-semigroups $J_{01}, J_{02}, J_{03}$ and $J_{04}$ are called fundamental ordered $J$-semigroups.

9. Regular pair of infinite order (2). In this section, the notations of elements $e_{n}, f_{n}, t, v$ and the notations of conditions (A)-( $\left(\mathrm{D}^{\prime}\right)$ are used just as is defined in $\S 7$. In $\S 7$, we divided the investigation of a regular pair of infinite order into two cases, and Case 1 was studied in that section. Now we study Case 2. Thus, in this section, we suppose that $(p, q)$ is a regular pair of infinite order such that $q \leqq p$ and $e_{2} \not \equiv e_{1} f_{1}\left(D_{E}\right)$.

Case 2 is divided into two subcases:

Case 2L: the subcase of Case 2 when $(p, q)$ is L-typed;

Case 2R: the subcase of Case 2 when $(p, q)$ is R-typed, and moreover Case $2 L$ is divided into two subcases:

Case 2L1: the subcase of Case $2 L$ when the condition (C) holds; 
Case 2L2: the subcase of Case 2L when (C) does not hold.

Now we consider Case 2L1. Thus we assume that $(p, q)$ is $L$ typed and satisfies (C). Then, by Lemma 12, the condition $\left(\mathrm{A}^{\prime}\right)$ holds. Hence $\left(e_{1} f_{1}\right) e_{2}=e_{1}\left(f_{1} e_{1}\right)=e_{1} f_{1}$, and so, since $e_{2} \not \equiv e_{1} f_{1}\left(D_{E}\right)$, we obtain $e_{2} f_{1}=e_{2}\left(e_{1} f_{1}\right) \neq e_{2}$. Hence (D) does not hold, and so, by Lemma 12, $\left(\mathrm{B}^{\prime}\right)$ does not hold. Hence $(\mathrm{A})$ and $\left(\mathrm{C}^{\prime}\right)$ hold. Moreover, by Lemma 11,

$$
e_{2}=e_{3}=e_{4}=\cdots \text {. }
$$

Since $(p, q)$ is $L$-typed, we have, by Lemma 3 ,

$$
t=e_{1} f_{1}, v=f_{1} e_{1}, e_{1} f_{1} e_{1}=e_{1} f_{1}, f_{1} e_{1} f_{1}=f_{1} e_{1} .
$$

Now we denote by $E$ the set consisting of elements $e_{2}, e_{1}, t, v, f_{1}$, $f_{2}, f_{3}, \cdots$.

We have $e_{1}<t$. In fact, otherwise, we would have $e_{1}=e_{1} f_{1}$ and so, by Lemma $10, e_{2}=e_{2} f_{1}$, which is a relation in (D). We have $f_{1}<f_{2}$. In fact, otherwise, we would have $f_{1}=f_{2}$ and so $e_{1} f_{1}=e_{1} f_{2}$, which is a relation in $\left(\mathrm{B}^{\prime}\right)$. We have $f_{n}<f_{n+1}$ for every $n \geqq 2$. In fact, otherwise, we would have $f_{n}=f_{n+1}$. Without loss of generality, we assume that $q p \leqq p q$. Then, by Corollary of Lemma $9, e_{n}=q^{n} p^{n}$ and $f_{n}=p^{n} q^{n}$. Hence, by (37), (A) and (A'), we would have

$$
\begin{aligned}
e_{2} & =e_{n}=q^{n} p^{n} q^{n} p^{n}=q^{n} f_{n} p^{n}=q^{n} f_{n+1} p^{n}=e_{n} f_{1} e_{n} \\
& =e_{2} f_{1} e_{2}=\left(e_{2} f_{1}\right)\left(f_{1} e_{2}\right)=\left(e_{1} f_{1}\right)\left(f_{1} e_{1}\right)=e_{1} f_{1}=t,
\end{aligned}
$$

which contradicts that $e_{2} \leqq e_{1}<t$. Thus the elements of $E$ are ordered by

$$
e_{2} \leqq e_{1}<t \leqq v \leqq f_{1}<f_{2}<f_{3}<\cdots .
$$

Using Lemma 10 and conditions $(\mathrm{A}),\left(\mathrm{A}^{\prime}\right),(\mathrm{C}),\left(\mathrm{C}^{\prime}\right)$, we can verify that the elements of $E$ are multiplied together just as in the table (34) in Example 5 in $\$ 8$.

Case $2 L 1$ is divided into two subcases:

Case 2L11: the subcase of Case $2 L 1$ when $q p \leqq p q$;

Case 2L12: the subcase of Case $2 L 1$ when $p q \leqq q p$.

Now we consider Case 2L11. Then $e_{n}=q^{n} p^{n}, f_{n}=p^{n} q^{n}$, and so, by $(\mathrm{C})$ and $\left(\mathrm{C}^{\prime}\right)$,

$$
q p^{3}=p^{2}, \quad q^{3} p=q^{2} .
$$

Moreover, by $e_{1} f_{1}=e_{1} f_{1} e_{1}$ and $f_{1} e_{1}=f_{1} e_{1} f_{1}$, we obtain

$$
p^{2} q=p^{2} q^{2} p, \quad q^{2} p=q^{2} p^{2} q \text {. }
$$


Now we consider the mappings $\varphi$ and $\psi$ of $E$ into itself which have been defined in $\S 8$, that is,

$$
\begin{gathered}
\varphi\left(e_{2}\right)=\varphi\left(e_{1}\right)=\varphi(t)=\varphi(v)=e_{2}, \varphi\left(f_{1}\right)=e_{1}, \\
\varphi\left(f_{2}\right)=t, \varphi\left(f_{3}\right)=f_{2}, \cdots ; \\
\psi\left(e_{2}\right)=v, \psi\left(e_{1}\right)=f_{1}, \psi(t)=\psi \\
\psi(v)=\psi\left(f_{1}\right)=f_{2}, \\
\psi\left(f_{2}\right)=f_{3}, \psi\left(f_{3}\right)=f_{4}, \cdots .
\end{gathered}
$$

Then, by (39) and (40), we can verify that

$$
q g=\varphi(g) q, \quad p g=\psi(g) p \text { for every } g \in E .
$$

Moreover, as is shown in $\S 8, \varphi$ and $\psi$ satisfy the conditions: (a)-(d) given there. Hence, if $l \geqq m+1$, then

$$
\begin{aligned}
\varphi^{l}(g) q^{m+1} p^{n+1} & =\varphi^{l}(g) q^{m} e_{1} p^{n}=\varphi^{l}(g) \varphi^{m}\left(e_{1}\right) q^{m} p^{n} \\
& =\varphi^{m}\left(\varphi^{l-m}(g) e_{1}\right) q^{m} p^{n}=\varphi^{m}\left(\varphi^{l-m}(g)\right) q^{m} p^{n}=\varphi^{l}(g) q^{m} p^{n} .
\end{aligned}
$$

Similarly, if $l \geqq m+1$, then we have

$$
\psi^{l}(g) p^{m+1} q^{n+1}=\psi^{l}(g) p^{m} q^{n} .
$$

Using these relations, we can verify that

$$
\begin{aligned}
& \left\{\begin{array}{l}
g q^{m} h q^{n}=g \varphi^{m}(h) q^{m+n}, \\
g q^{m} h=g \varphi^{m}(h) q^{m}, \\
g q^{m} h p^{n}= \begin{cases}g \varphi^{m}(h) q^{m-n} & \text { if } m>n, \\
g \varphi^{m}(h) & \text { if } m=n, \\
g \varphi^{m}(h) p^{n-m} & \text { if } m<n .\end{cases}
\end{array}\right. \\
& \left\{\begin{array}{l}
g p^{m} h p^{n}=g \psi^{m}(h) p^{m+n}, \\
g p^{m} h=g \psi^{m}(h) p^{m}, \\
g p^{m} h q^{n}= \begin{cases}g \psi^{m}(h) p^{m-n} & \text { if } m>n, \\
g \psi^{m}(h) & \text { if } m=n, \\
g \psi^{m}(h) q^{n-m} & \text { if } m<n .\end{cases}
\end{array}\right.
\end{aligned}
$$

Now we denote by $J^{*}$ the set consisting of elements of the forms $g q^{n}, g p^{n}$ or $g$ with $g \in E$ and natural number $n$. By (42) and (43), we see that $J^{*}$ is a subsemigroup, which is then clearly the subsemigroup generated by regular pair $(p, q)$.

Since $q=e_{1} q$, we have

$$
v q=f_{1} q .
$$

By (44) and $e_{2} q^{2}=q^{2}=e_{1} q^{2}$, we have

$$
e_{2} q^{n}=e_{1} q^{n}, \quad v q^{n}=f_{1} q^{n} \text { for } n \geqq 2 \text {. }
$$


Since $t p=q p^{2} q p=q p^{2}=q^{2} p^{3}=e_{2} p \leqq e_{1} p \leqq t p$, we have

$$
e_{2} p=e_{1} p=t p \text {. }
$$

If $n \geqq 2$, then $f_{n} p^{n}=p^{n} q^{n} p^{n}=p^{n}=q p^{n+1}=q^{2} p^{n+2}=e_{2} p^{n}$, and so,

$$
e_{2} p^{n}=e_{1} p^{n}=t p^{n}=v p^{n}=f_{1} p^{n}=\cdots=f_{n} p^{n} \quad \text { for } n \geqq 2 .
$$

We have $f_{n} q^{m+1}<e_{2} q^{m}$, for, otherwise, we would have

$$
q^{m+n+1}=q^{n} f_{n} q^{m+1} \geqq q^{n} e_{2} q^{m}=\varphi^{n}\left(e_{2}\right) q^{m+n}=q^{m+n},
$$

which contradicts Lemma 9. Therefore we also have $f_{n} q<e_{2}$. We have $e_{1} q^{n}<t q^{n}$, for, otherwise, we would have

$$
e_{n}=e_{1} q^{n} p^{n} \geqq t q^{n} p^{n}=t e_{n}=t,
$$

which contradicts (38). Similarly we obtain $f_{n} q^{m}<f_{n+1} q^{m}$. We have $f_{n} p^{m}<e_{2} p^{m+1}$, for, otherwise, we would have

$$
f_{n} f_{m} q=f_{n} p^{m} q^{m+1} \geqq e_{2} p^{m+1} q^{m+1}=e_{2} f_{m+1} \geqq e_{2},
$$

which contradicts $f_{l} q<e_{2}$. If $m \geqq n$, then we have $f_{m} p^{n}<f_{m+1} p^{n}$, for, otherwise, we would have

$$
f_{m}=f_{m} p^{n} q^{n} \geqq f_{m+1} p^{n} q^{n}=f_{m+1},
$$

which contradicts (38). Thus the elements of $J^{*}$ are ordered by

$$
\begin{aligned}
\cdots & <e_{2} q^{2}<t q^{2} \leqq v q^{2}<f_{2} q^{2}<f_{3} q^{2}<\cdots<e_{2} q \leqq e_{1} q<t q \\
& \leqq v q<f_{2} q<f_{3} q<\cdots<e_{2} \leqq e_{1}<t \leqq v \leqq f_{1}<f_{2}<f_{3}<\cdots \\
& <t p \leqq v p \leqq f_{1} p<f_{2} p<f_{3} p<\cdots<f_{2} p^{2}<f_{3} p^{2}<\cdots
\end{aligned}
$$

Lemma 15. In this Case 2L11, the following four conditions are equivalent to each other:

$$
v q=f_{1} p, v=f_{1}, e_{2}=e_{1}, e_{2} q=e_{1} q .
$$

Proof. If $v p=f_{1} p$, then $v=v p q=f_{1} p q=f_{1}$. If $v=f_{1}$, then $e_{2}=q v p=q f_{1} p=e_{1}$. If $e_{2}=e_{1}$, then clearly $e_{2} q=e_{1} q$. If $e_{2} q=e_{1} q$, then $v p=p e_{2} q p=p e_{1} q p=p=f_{1} p$.

Lemma 16. In this Case 2L11, all the following conditions are equivalent to each other:

$$
\cdots, t q^{3}=v q^{3}, t q^{2}=v q^{2}, t q=v q, t=v, t p=v p .
$$

Proof. If $t=v$, then clearly $t q^{n}=v q^{n}$ and $t p=v p$. If $t p=v p$, then $t=t p q=v p q=v$. Similarly, if $t q^{n}=v q^{n}$ for some $n$, then $t=v$.

Now we investigate Case $2 L 11$ by dividing into several subcases. 
$1^{\circ}$. Subcase of Case $2 L 11$ when $e_{2} \neq e_{1}, t \neq v$.

By Lemmas 15 and 16, all the elements of $J^{*}$ written in (48) are different. We consider mapping of $J_{11 L}$ of Example 5 in $\S 8$ into $I^{*}$ :

$$
(n, g) \rightarrow \begin{cases}g p^{n} & \text { if } n>0, \\ g & \text { if } n=0 \\ g q^{-n} & \text { if } n<0\end{cases}
$$

By (36) in $\S 8$, this mapping is one-to-one and onto. Moreover it is order-preserving. Furthermore, comparing (35) in $\S 8$ to (42) and (43), we see that this mapping is an isomorphism. Thus $J^{*}$ is isomorphic to $J_{11 L}$. We remark, by the above isomorphism, $\left(-1, e_{1}\right)$ and $\left(1, f_{1}\right)$ are mapped into $q$ and $p$, respectively.

$2^{\circ}$. Subcase of Case $2 L 11$ when $e_{2}=e_{1}, t \neq v$.

By Lemmas 15 and $16, J^{*}$ is isomorphic to $J_{21 L}$ of Example 9 in $\S 8$.

$3^{\circ}$. Subcase of Case $2 L 11$ when $e_{2} \neq e_{1}, t=v$.

By Lemmas 15 and $16, J^{*}$ is isomorphic to $J_{31}$ of Example 13 in $\S 8$.

$4^{\circ}$. Subcase of Case $2 L 11$ when $e_{2}=e_{1}, t=v$.

By Lemmas 15 and $16, J^{*}$ consists of elements

$\cdots<e_{2} q^{2}<t q^{2}<f_{2} q^{2}<f_{3} q^{2}<\cdots<e_{2} q<t q<f_{2} q<f_{3} q<\cdots$

$\cdots<e_{2}<t<f_{2}<f_{3}<\cdots<t p<f_{2} p<f_{3} p<\cdots<f_{2} p^{2}$

$$
<f_{3} p^{2}<\cdots \text {. }
$$

We can verify that the mapping of $J_{01}$ into $J^{*}$ defined by

$$
(m, n) \rightarrow \begin{cases}e_{2} q^{n} & \text { if } m=0, n>0, \\ e_{2} & \text { if } m=n=0, \\ t q^{n-1} & \text { if } m=1, n>1, \\ t & \text { if } m=n=1, \\ t p & \text { if } m=1, n=0, \\ f_{m} q^{n-m} & \text { if } m \geqq 2, m<n, \\ f_{m} & \text { if } m \geqq 2, m=n, \\ f_{m} p^{m-n} & \text { if } m \geqq 2, m>n \geqq 0\end{cases}
$$

is an isomorphism onto $J^{*}$. 
Similarly we can discuss Case $2 L 12$.

Now we consider Case 2L2. In this case, (C) does not hold, and so, by Lemma 12, also $\left(\mathrm{A}^{\prime}\right)$ does not hold. Hence $(\mathrm{B})$ and $\left(\mathrm{D}^{\prime}\right)$ hold, and so, by Lemma 12, also $\left(\mathrm{B}^{\prime}\right)$ and $(\mathrm{D})$ hold. Thus we can argue in a similar way as in Case $2 L 1$.

We can discuss Case $2 R$ in a similar way.

THEOREM 5. Using the notations given in (12), let $(p, q)$ be a regular pair of infinite order such that $q \leqq p$ and $e_{2} \equiv e_{1} f_{1}\left(D_{E}\right)$, let $J^{*}$ be the subsemigroups generated by $(p, q)$, and let $J_{01}-J_{24 R}$ be the ordered $J$-semigroups given in $\S 8$.

(a) If $q p \leqq p q, e_{2}=e_{1}, t=v$, then $J^{*}$ is isomorphic to $J_{01}$;

(b) if $p q \leqq q p, e_{2}=e_{1}, t=v$, then $J^{*}$ is isomorphic to $J_{02}$;

(c) if $p q \leqq q p, f_{1}=f_{2}, t=v$, then $J^{*}$ is isomorphic to $J_{03}$;

(d) if $q p \leqq p q, f_{1}=f_{2}, t=v$, then $J^{*}$ is isomorphic to $J_{04}$;

(e) if $(p, q)$ is L-typed, $q p \leqq p q, f_{1} \leqq f_{2} e_{1}, e_{2} \neq e_{1}, t \neq v$, then $J^{*}$ is isomorphic to $J_{11 L}$;

(f) if $(p, q)$ is L-typed, $p q \leqq q p, f_{1} \leqq f_{2} e_{1}, e_{2} \neq e_{1}, t \neq v$, then $J^{*}$ is isomorphic to $J_{12 L}$;

(g) if $(p, q)$ is L-typed, $p q \leqq q p, f_{2} e_{1}<f_{1}, f_{1} \neq f_{2}, t \neq v$, then $J^{*}$ is isomorphic to $J_{13 L}$;

(h) if $(p, q)$ is L-typed, $q p \leqq p q, f_{2} e_{1}<f_{1}, f_{1} \neq f_{2}, t \neq v$, then $J^{*}$ is isomorphic to $J_{14 L}$;

(i) if $(p, q)$ is L-typed, $q p \leqq p q, f_{1} \leqq f_{2} e_{1}, e_{2}=e_{1}, t \neq v$, then $J^{*}$ is isomorphic to $J_{212}$;

(j) if $(p, q)$ is L-typed, $p q \leqq q p, f_{1} \leqq f_{2} e_{1}, e_{2}=e_{1}, t \neq v$, then $J^{*}$ is isomorphic to $J_{22 L}$;

(k) if $(p, q)$ is L-typed, $p q \leqq q p, f_{2} e_{1}<f_{1}, f_{1}=f_{2}, t \neq v$, then $J^{*}$ is isomorphic to $J_{23 L}$;

(1) if $(p, q)$ is L-typed, $q p \leqq p q, f_{2} e_{1}<f_{1} f_{1}=f_{2}, t \neq v$, then $J^{*}$ is isomorphic to $J_{24 L}$;

(m) if $q p \leqq p q, e_{2} \neq e_{1}, t=v$, either $(p, q)$ is L-typed and $f_{1} \leqq$ $f_{2} e_{1}$ or $(p, q)$ is R-typed and $f_{1} \leqq e_{1} f_{2}$, then $J^{*}$ is isomorphic to $J_{31}$;

(n) if $p q \leqq q p, e_{2} \neq e_{1}, t=v$, either $(p, q)$ is L-typed and $f_{1} \leqq$ $f_{2} e_{1}$ or $(p, q)$ is $R$-typed and $f_{1} \leqq e_{1} f_{2}$, then $J^{*}$ is isomorphic to $J_{32}$;

(o) if $p q \leqq q p, f_{1} \neq f_{2}, t=v$, either $(p, q)$ is L-typed and $f_{2} e_{1}<f_{1}$ or $(p, q)$ is $R$-typed and $e_{1} f_{2}<f_{1}$, then $J^{*} i$ s isomorphic to $J_{33}$;

(p) if $q p \leqq p q, f_{1} \neq f_{2}, t=v$, either $(p, q)$ is L-typed and $f_{2} e_{1}<f_{1}$ or $(p, q)$ is $R$-typed and $e_{1} f_{2}<f_{1}$, then $J^{*}$ is isomorphic to $J_{34}$;

(q) if $(p, q)$ is $R$-typed, $p q \leqq q p, f_{1} \leqq e_{1} f_{2}, e_{2} \neq e_{1}, t \neq v$, then $J^{*}$ is isomorphic to $J_{11 R}$;

(r) if $(p, q)$ is $R$-typed, $q p \leqq p q, f_{1} \leqq e_{1} f_{2}, e_{2} \neq e_{1}, t \neq v$, then $J^{*}$ is isomorphic to $J_{12 R}$; 
(s) if $(p, q)$ is $R$-typed, $q p \leqq p q, e_{1} f_{2}<f_{1}, f_{1} \neq f_{2}, t \neq v$, then $J^{*}$ is isomorphic to $J_{13 R}$;

(t) if $(p, q)$ is R-typed, $p q \leqq q p, e_{1} f_{2}<f_{1}, f_{1} \neq f_{2}, t \neq v$, then $J^{*}$ is isomorphic to $J_{14 R}$;

(u) if $(p, q)$ is R-typed, $p q \leqq q p, f_{1} \leqq e_{1} f_{2}, e_{2}=e_{1}, t \neq v$, then $J^{*}$ is isomorphic to $J_{21 R}$;

(v) if $(p, q)$ is $R$-typed, $q p \leqq p q, f_{1} \leqq e_{1} f_{2}, e_{2}=e_{1}, t \neq v$, then $J^{*}$ is isomorphic to $J_{22 R}$;

(w) if $(p, q)$ is $R$-typed, $q p \leqq p q, e_{1} f_{2}<f_{1}, f_{1}=f_{2}, t \neq v$, then $J^{*}$ is isomorphic to $J_{23 R}$;

(x) if $(p, q)$ is $R$-typed, $p q \leqq q p, e_{1} f_{2}<f_{1}, f_{1}=f_{2}, t \neq v$, then $J^{*}$ is isomorphic to $J_{24 R}$.

CoRollary. Under the assumptions of Theorem 5, $J^{*}$ contains a subsemigroup which is isomorphic to one of the fundamental ordered J-semigroups.

$\S 10$. Applications. A semigroup $S$ is called an inverse semigroup if every element of $S$ is regular and each pair of idempotents of $S$ commute (Munn and Penrose [6]). It can be seen that every subsemigroup of an inverse semigroup $S$ in which every element is regular is an inverse subsemigroup. Hence, by Corollary 2 of Lemma 5 , for a regular pair $(p, q)$ of $S$, the subsemigroup generated by $(p, q)$ is an inverse subsemigroup. Now we see that, except $I_{0}, J_{01}, J_{02}, J_{03}$ and $J_{04}$, all ordered semigroups given in examples in $\S \S 4,6$ and 8 are not inverse semigroups. Hence we have

THEOREM 6. Let $(p, q)$ be a non-idempotent regular pair of an ordered inverse semigroup. Then the subsemigroup generated by $(p, q)$ is isomorphic to either the additive ordered group $I_{0}$ of integers or one of the fundamental ordered J-semigroups.

Evidently fundamental ordered $J$-semigroups are not commutative. Hence we have

THEOREM 7. Let $(p, q)$ be a non-idempotent regular pair of an ordered commutative semigroup. Then the subsemigroup generated by $(p, q)$ is isomorphic to the additive ordered group $I_{0}$ of integers.

\section{REFERENCES}

1. N. G. Alimov, On ordered semigroups, Izvestiya Akad. Nauk SSSR., 14 (1950), 569-576 (Russian).

2. A. H. Clifford, Totally ordered commutative semigroups, Bull. Amer. Math. Soc., 64 (1958), 305-316. 
3. J. A. Green, On the structure of semigroups, Ann. of Math., 54 (1951), 163-172.

4. E. S. Lyapin, Semigroups, 1960 (Russian).

5. D. D. Miller and A. H. Clifford, Regular D-classes in semigroups, Trans. Amer. Math. Soc., 82 (1956), 270-280.

6. W. D. Munn and R. Penrose, A note on inverse semigroups, Proc. Cambridge Phil. Soc., 51 (1955), 396-399.

7. G. B. Preston, Inverse semigrougs, J. London Math. Soc., 29 (1954), 396-403.

8. —, Representations of inverse semigroups, J. London Math. Soc., 29 (1954), 411-419.

9. T. Saitô, Note on semigroups having no minimal ideals, Bull. Tokyo Gakugei Univ., 9 (1958), 13-16.

10. - Ordered idempotent semigroups, J. Math. Soc. Japan, 14 (1962), 150-169. 11. M. G. Thierrin, Sur une condition nécessaire et suffisante pour qu'un semigroupe soit un groupe, C. R. Acad. Paris, 232 (1951), 376-378.

TOKYO GAKUGEI UNIVERSITY, JAPAN 



\section{PACIFIC JOURNAL OF MATHEMATICS}

\section{EDITORS}

\section{RalPh S. Phillips}

Stanford University

Stanford, California

M. G. Arsove

University of Washington

Seattle 5, Washington
J. Dugundji

University of Southern California Los Angeles 7, California

Lowell J. Paige

University of California

Los Angeles 24, California

\section{ASSOCIATE EDITORS}
E. F. BECKENBACH
D. DERRY
H. L. ROYDEN
E. G. STRAUS
T. M. CHERRY
M. OHTSUKA
E. SPANIER
F. WOLF

\section{SUPPORTING INSTITUTIONS}

\author{
UNIVERSITY OF BRITISH COLUMBIA \\ CALIFORNIA INSTITUTE OF TECHNOLOGY \\ UNIVERSITY OF CALIFORNIA \\ MONTANA STATE UNIVERSITY \\ UNIVERSITY OF NEVADA \\ NEW MEXICO STATE UNIVERSITY \\ OREGON STATE UNIVERSITY \\ UNIVERSITY OF OREGON \\ OSAKA UNIVERSITY \\ UNIVERSITY OF SOUTHERN CALIFORNIA
}

STANFORD UNIVERSITY

UNIVERSITY OF TOKYO

UNIVERSITY OF UTAH

WASHINGTON STATE UNIVERSITY

UNIVERSITY OF WASHINGTON

AMERICAN MATHEMATICAL SOCIETY

CALIFORNIA RESEARCH CORPORATION SPACE TECHNOLOGY LABORATORIES

NAVAL ORDNANCE TEST STATION

Printed in Japan by International Academic Printing Co., Ltd., Tokyo Japan 


\section{Pacific Journal of Mathematics}

\section{Vol. 13, No. 1 \\ March, 1963}

Frantz Woodrow Ashley, Jr., A cone of super-(L) functions............. 1

Earl Robert Berkson, Some metrics on the subspaces of a Banach space....

Felix Earl Browder and Walter Strauss, Scattering for non-linear wave

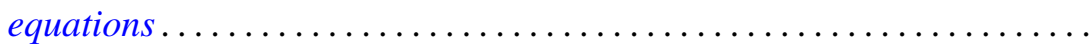

Edmond Darrell Cashwell and C. J. Everett, Formal power series ..........

Frank Sydney Cater, Continuous linear functionals on certain topological

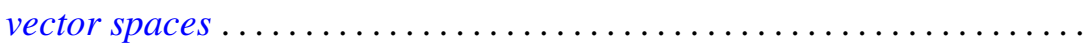

John Douglas Dixon, General group extensions ....................

Robert Pertsch Gilbert, On harmonic functions of four variables with

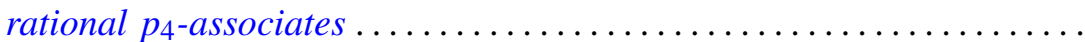

Irving Leonard Glicksberg, On convex hulls of translates ..............

Simon Hellerstein, On a class of meromorphic functions with deficient zeros

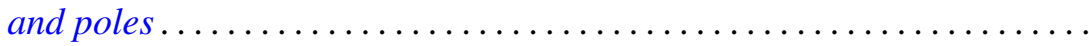

Donald William Kahn, Secondary cohomology operations which extend the

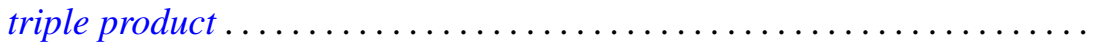

G. K. Leaf, A spectral theory for a class of linear operators .............

R. Sherman Lehman, Algebraic properties of the composition of solutions of partial differential equations ........................... 157

Joseph Lehner, On the generation of discontinuous groups ............. 169

S. P. Lloyd, On certain projections in spaces of continuous functions ...... 171 Fumi-Yuki Maeda, Generalized spectral operators on locally convex spaces ..................................

Donald Vern Meyer, $E^{3}$ modulo a 3-cell

William H. Mills, An application of linear programming to permutation groups.

Richard Scott Pierce, Centers of purity in abelian groups

Christian Pommerenke, On meromorphic starlike functions ...

Zalman Rubinstein, Analytic methods in the study of zeros of

polynomials...

B. N. Sahney, On the Nörlund summability of Fourier series

Tôru Saitô, Regular elements in an ordered semigroup . .

Lee Meyers Sonneborn, Level sets on spheres...........

Charles Andrew Swanson, Asymptotic estimates for limit point

problems .

Lucien Waelbroeck, On the analytic spectrum of Arens . .

Alvin (Murray) White, Singularities of a harmonic function of three

variables given by its series development .............

Kōichi Yamamoto, Decomposition fields of difference sets ...

Chung-Tao Yang, On the action of $\mathrm{SO}(3)$ on a cohomology manifold... 